\title{
A hybrid origin for the Qianshan A-type granite, northeast China: Geochemical and $\mathrm{Sr}-\mathrm{Nd}-\mathrm{Hf}$ isotopic evidence
}

\author{
Jin-Hui Yang ${ }^{\mathrm{a}, *}$, Fu-Yuan $\mathrm{Wu}^{\mathrm{a}}$, Sun-Lin Chung ${ }^{\mathrm{b}}$, Simon A. Wilde ${ }^{\mathrm{c}}$, Mei-Fei Chu ${ }^{\mathrm{b}}$ \\ a State Key Laboratory of Lithosphere Evolution, Institute of Geology and Geophysics, Chinese Academy of Sciences, P.O. Box 9825, \\ Beijing 100029, China \\ b Department of Geosciences, National Taiwan University, Taipei 106, Taiwan \\ c Department of Applied Geology, Curtin University of Technology, P.O. Box U1987, Perth, Western Australia 6845, Australia
}

Received 2 March 2005; accepted 31 October 2005

Available online 23 January 2006

\begin{abstract}
Major and trace element, whole rock $\mathrm{Sr}$ and $\mathrm{Nd}$ isotope and zircon $\mathrm{Hf}$ isotope data are reported for a suite of A-type granites and mafic microgranular enclaves from the Early Cretaceous (126 $\pm 2 \mathrm{Ma}$ ) Qianshan pluton, Liaodong Peninsula, northeast China, with the aim of investigating the sources and petrogenesis of A-type granites. The Qianshan pluton includes hornblende alkali-feldspar granite, graphic biotite granite and mafic microgranular enclaves. The hornblende alkali-feldspar granites have high $\mathrm{SiO}_{2}, \mathrm{Fe}_{2} \mathrm{O}_{3} /$ $\mathrm{MgO}, \mathrm{K}_{2} \mathrm{O}+\mathrm{Na}_{2} \mathrm{O}, \mathrm{Rb}, \mathrm{Zr}$ and LREE contents and low $\mathrm{Ba}$ and $\mathrm{Sr}$ concentrations with strongly negative Eu anomalies. Their high $\mathrm{Rb} / \mathrm{Sr}\left({ }^{87} \mathrm{Rb} /{ }^{86} \mathrm{Sr}=16.76-24.15\right)$ and initial ${ }^{87} \mathrm{Sr} /{ }^{86} \mathrm{Sr}$ ratios $(0.7215$ to 0.7283$)$, negative $\varepsilon_{\mathrm{Nd}}(t)$ values $(-14.1$ to -16.5$)$ and zircon $\varepsilon_{\mathrm{Hf}}(t)$ values $(-18.9$ to -11.5$)$ indicate they were mainly derived from a crustal source, but with involvement of high $\varepsilon_{\mathrm{Nd}}(t)$ and $\varepsilon_{\mathrm{Hf}}(t)$ materials. Graphic biotite granites have similar geochemical features and $\mathrm{Sr}-\mathrm{Nd}-\mathrm{Hf}$ isotopic compositions to enclaves, indicating they were the result of crystal fractionation of evolved mafic magmas, but with involvement of low $\varepsilon_{\mathrm{Nd}}(t)$ and $\varepsilon_{\mathrm{Hf}}(t)$ materials. The mafic enclaves have an igneous texture and contain acicular apatite, suggesting quenching of mafic magmas that have co-mingled with the host granites. They have low initial ${ }^{87} \mathrm{Sr} /{ }^{86} \mathrm{Sr}$ ratios $(0.7097-0.7148)$, negative $\varepsilon_{\mathrm{Nd}}(t)(-14.5$ to -11.9$)$ and zircon $\varepsilon_{\mathrm{Hf}}(t)(-17.1$ to -6.9$)$ values, and are enriched in LILEs and LREEs and depleted in HFSEs. When coupled with the high $\mathrm{MgO}$ ( $\mathrm{Mg \#}$ up to 54), this indicates derivation from an enriched lithospheric mantle source, but contaminated by crustal materials.

Geochemical and Sr-, Nd- and zircon Hf-isotopic compositions rule out simple crystal-liquid fractionation or restite unmixing as the major genetic link between enclaves and host rocks. Instead, magma mixing of mantle-derived mafic and crustal-derived magmas, coupled with crystal fractionation, is compatible with the data. This example shows that at least some A-type granites formed through a complex process involving mantle- and crustal-derived magma mixing, crystal fractionation and infracrustal melting.
\end{abstract}

(C) 2005 Elsevier B.V. All rights reserved.

Keywords: A-type granite; Magma mixing; Sr-Nd isotopes; Mafic microgranular enclaves; Zircon Hf isotopes

\footnotetext{
* Corresponding author. Tel.: +86 10 62007900; fax: +86 10 62010846.

E-mail address: jinhui@mail.igcas.ac.cn (J.-H. Yang).
}

\section{Introduction}

"A-type" granites commonly occur in post-orogenic or intraplate tectonic settings (Eby, 1990, 1992; Black and Liegeois, 1993) and provide significant information 
on post-collisional/intraplate extensional magmatic processes within the continental lithosphere and their contribution to the build-up of the upper continental crust (Turner et al., 1992; Mushkin et al., 2003). However, several petrogenetic schemes have been proposed for their origin: (1) direct fractionation products of mantlederived alkaline basalts (e.g., Turner et al., 1992; Litvinovsky et al., 2002; Mushkin et al., 2003); (2) low degrees of partial melting of F- and/or Cl-enriched dry, lower crustal granulitic residue from which a granitoid melt was previously extracted (e.g., Collins et al., 1982; Clemens et al., 1986; King et al., 1997); (3) lowpressure melting of calc-alkaline rocks at upper crustal levels (Skjerlie and Johnston, 1993; Patiño Douce, 1997); and (4) hybridization between anatectic granitic and mantle-derived mafic magmas (Bédard, 1990; Kerr and Fryer, 1993; Wickham et al., 1996; Mingram et al., 2000). An important and fundamental question is whether such granitoids are exclusively crustally derived or whether they require significant involvement of mantle materials; or even whether they can be generated directly by fractionation of basalt.

Mafic microgranular enclaves (MMEs of Didier and Barbarin, 1991) occur in nearly all granites and have been the focus of many studies (see reviews by Vernon, 1984; Didier and Barbarin, 1991; Bonin, 2004). Although MMEs may have originated in many different ways and from different sources, there has been a general consensus that textural criteria allow recognition of a class of enclaves that formed by mingling of an externally derived magma with granitic magma while it was still mobile (Vernon, 1984; Vernon et al., 1988); these can appropriately be termed magmatic enclaves (Wiebe et al., 1997). In spite of partial reequilibration, enclaves commonly have isotopic compositions that are distinct from the enclosing granite and support their formation from mantle-derived magmas (Holden et al., 1987; Yang et al., 2004b). However, MMEs commonly occur in I- and S-type granites and are rare in A-type granites (Didier and Barbarin, 1991), with only a few cases reported by Bédard (1990) and Wiebe et al. (1997). We have identified that MMEs in the Early Cretaceous A-type complex of the Liaodong Peninsula, northeastern China possess distinct isotopic characteristics that may be used to argue for magma mixing in the origin of certain A-type granites. In this paper we: (1) present new geochemical, $\mathrm{Sr}$ and $\mathrm{Nd}$ and in situ zircon Hf isotopic data for MMEs and their host A-type granites from the Qianshan pluton, (2) use these tracers to constrain the sources and petrogenesis of the A-type granites, and (3) discuss the role of magma mixing in the overall origin of A-type granites.

\section{Geological setting of the Qianshan pluton}

\subsection{Regional geological setting}

East China is composed of the Xing'an-Mongolia (Xing-Meng) Orogenic belt in the north, the North China Craton (NCC) in the centre and the Dabie-Sulu ultrahigh-pressure orogenic belt in the south and east (Fig. 1a, Wang and Mo, 1996).

The Liaodong Peninsula, located in the eastern segment of the NCC (Fig. 1a), consists of Archean to Paleoproterozoic basement rocks overlain by unmetamorphosed Mesoproterozoic to Paleozoic sediments and Mesozoic to Cenozoic sedimentary and volcanic rocks (Zhao et al., 2001). Early Archean basement rocks, which range in age from 3.85 to $3.2 \mathrm{Ga}$, have been reported near Anshan, north of the Liaodong Peninsula (Fig. 1, Liu et al., 1992; Song et al., 1996). In the Liaodong Peninsula, deformed late Archean gneisses include diorite, tonalite and granodiorite that were emplaced at about $2500 \mathrm{Ma}$ (Lu et al., 2004). In the Paleoproterozoic, the Liaohe Group was deposited and then metamorphosed during the $\sim 1.9$ Ga collisional event in the Liaodong Peninsula (Zhao et al., 2001). Subsequently, the Liaodong Peninsula was covered by a thick sequence of Meso- to Neoproterozoic and Paleozoic sediments (Wang and Mo, 1996). Paleozoic diamond-bearing kimberlites (LBGMR, 1986) and Cenozoic gabbros and basalts have also been found here.

In the Liaodong Peninsula, about $20,000 \mathrm{~km}^{2}$ of intrusive rocks have been identified along with minor volcanic rocks (LBGMR, 1986) (Fig. 1b). According to our recent work, these intrusions can be divided into three groups: (1) Late Triassic (238-212 Ma) syenites, monzogranites and gabbros (Yang et al., 2004a), (2) Jurassic (180-153 Ma) tonalite, diorite, and two-mica granites that have experienced ductile deformation (Wu et al., 2005b), and (3) Early Cretaceous (130-106 Ma) diorite, granodiorite, monzogranite and A-type granite (Yang et al., 2004b; Wu et al., 2005a). Coeval with the formation of Early Cretaceous I- and A-type granitoids was the exhumation of metamorphic core complexes, indicating that these granitoids were emplaced in an intra-plate extensional environment of regional extent (Wu et al., 2005a).

\subsection{Geology and petrography of the Qianshan pluton}

The Qianshan intrusion (Fig. 1c) is one of several Atype plutons located in the Liaodong Peninsula. It extends E-W and was intruded into Archean supracrustal rocks and granites, the Paleoproterozoic Liaohe Group 


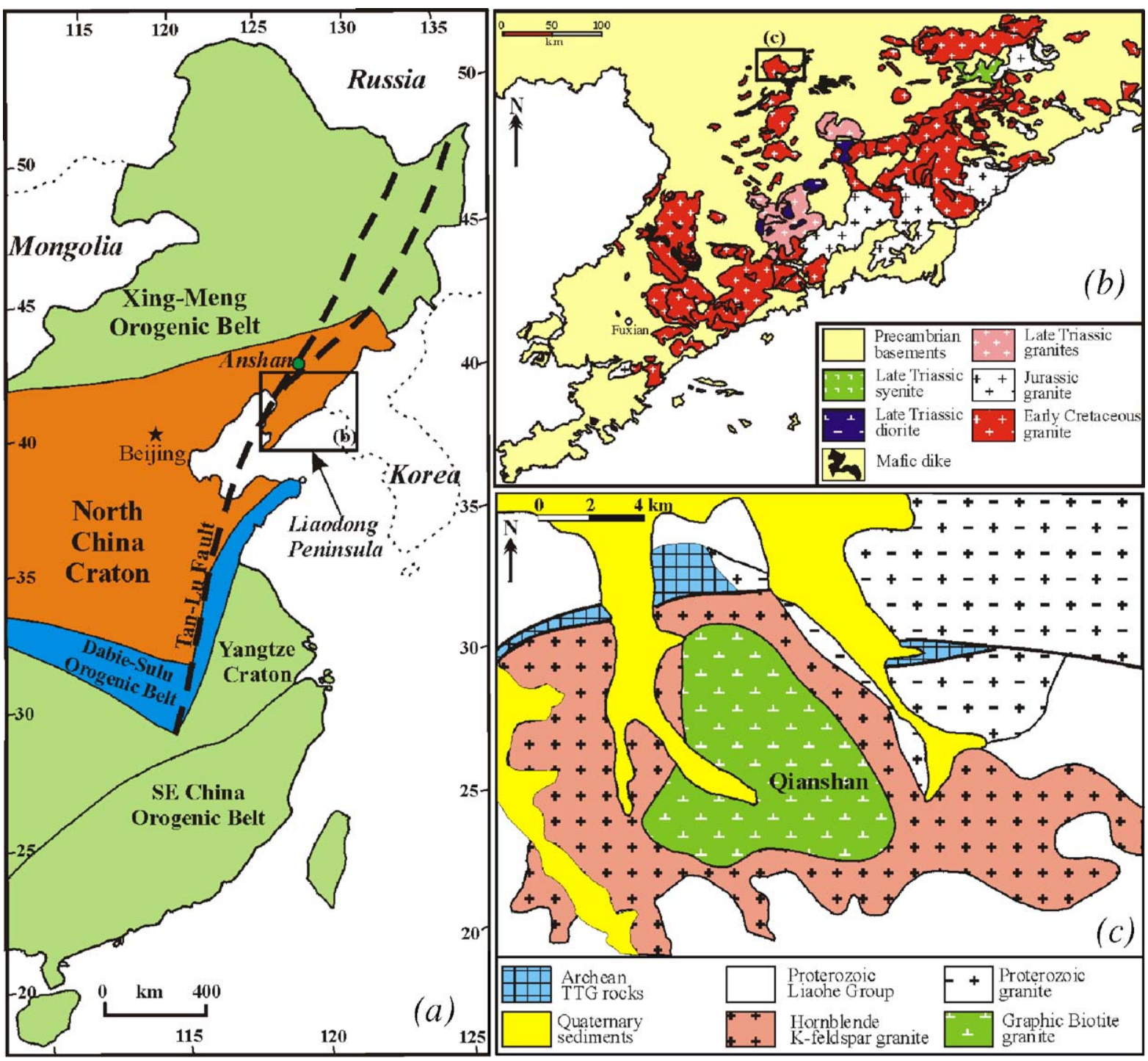

Fig. 1. (a) Simplified geological map of eastern China, showing major tectonic units; (b) geological map showing distribution of Mesozoic intrusions in the Liaodong Peninsula; and (c) geological map of the Qianshan pluton in the Liaodong Peninsula.

and Neoproterozoic sandstones. LA-ICP-MS zircon U$\mathrm{Pb}$ dating indicates that the emplacement age of the Qianshan granitoids is $126 \pm 2 \mathrm{Ma}$ (Wu et al., 2005a).

The pluton consists of two main phases that are texturally distinct. The hornblende alkali-feldspar granite (hornblende granite) is orange to red-brown and coarse-grained, consisting of $60 \%$ subhedral alkali feldspar, $5 \%$ plagioclase, $30 \%$ anhedral quartz, $1-5 \%$ hornblende and $<1 \%$ accessory minerals, including zircon and allanite. The graphic biotite granite (biotite granite) is orange to white and medium- to coarse-grained, consisting of $60 \%$ subhedral alkali feldspar, $0-5 \%$ plagioclase, $32 \%$ anhedral quartz, $1-5 \%$ biotite and $<1 \%$ accessory minerals, including zircon and apatite. This granite contains either individual xenocrysts (mantled plagioclases) or small, fine-grained aggregates (e.g., quartz/K-feldspar oikocrysts enclosing numerous small crystals of hornblende and plagioclase).

All granites contain ubiquitous mafic microgranular enclaves. The enclaves appear circular or lenticular in section, with sharp contacts, locally featuring chilled margins or thin biotite-rich reaction rims (Fig. 2). Interaction (or hybridization) between the mafic enclaves and host felsic magma is evident from the presence of gradational contacts, suggesting that magma mixing/ mingling occurred. Disaggregation of mafic enclaves yields heterogeneous hybrid rocks containing wispy schlieren and clots of fine-grained mafic material that 

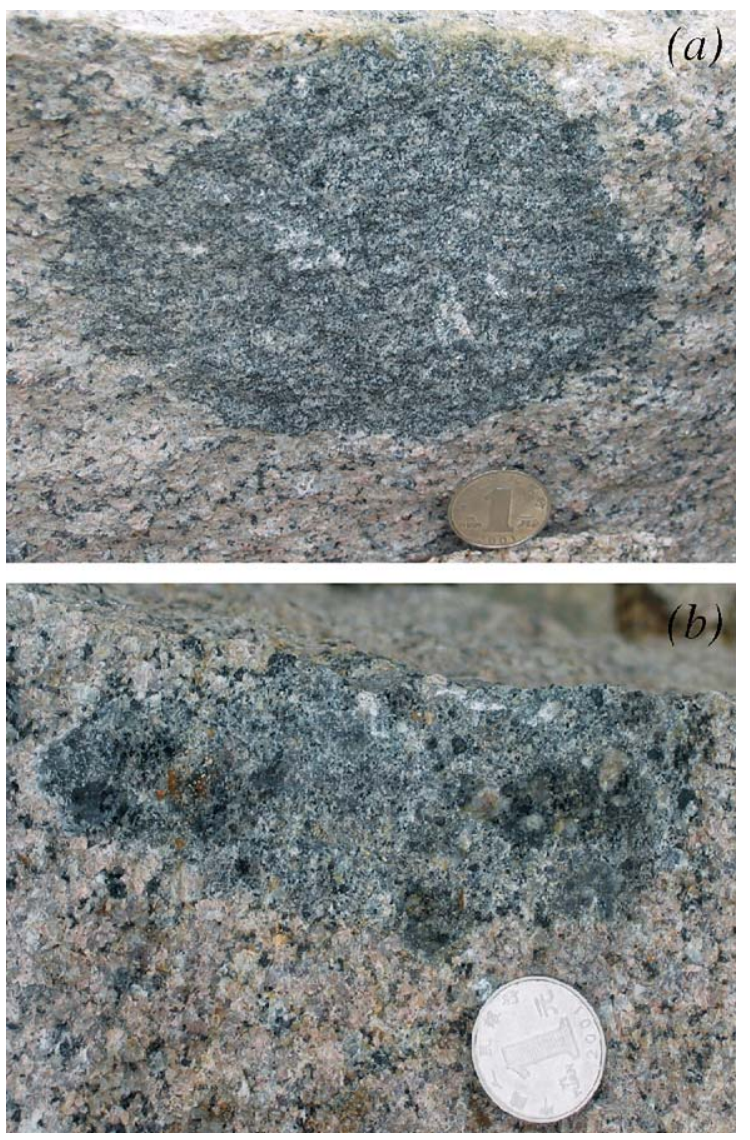

Fig. 2. Examples (a and b) of mafic microgranular enclaves (MMEs) occurring within A-type granites in the Qianshan pluton. The diameter of the 1 yuan coin is $25 \mathrm{~mm}$.

are heterogeneously distributed throughout the granite. The rocks range from dioritic to monzogranite in composition and are composed of $20-30 \%$ hornblende, 30 $40 \%$ alkali feldspar, $20-30 \%$ plagioclase, $5 \%$ biotite and $<1 \%$ acicular apatite. Plagioclase occurs both as small euhedral laths and as larger mantled crystals, commonly enclosing small rounded hornblende grains. Hornblende is typically subhedral and forms monomineralic clots with lobate outlines or small anhedral crystals, interstitial to plagioclase. Both K-feldspar and quartz are interstitial and poikilitic. Some K-feldspar occurs as megacrysts (Fig. 2b). Apatite is a common accessory and tends to form long prismatic, acicular crystals.

\section{Analytical methods}

\subsection{Major and trace elements}

After petrographic examination, a total of 29 fresh rock samples were selected, crushed, and powdered in an agate mill. Major elements were determined by Xray fluorescence techniques on fused glass beads using Rigaku ${ }^{\circledR}$ RIX-2000 spectrometers at the National Taiwan University. The analytical procedures were the same as those described by Lee et al. (1997) and Wang et al. (2004), yielding analytical uncertainties better than $\pm 5 \%(2 \sigma)$ for all major elements. The fused glass beads were powdered and dissolved using an $\mathrm{HF} / \mathrm{HNO}_{3}(1: 1)$ mixture in screw-top Teflon beakers for $12 \mathrm{~h}$ at $\sim 100{ }^{\circ} \mathrm{C}$, followed by evaporation to dryness, then refluxing in $7 \mathrm{~N} \mathrm{HNO}_{3}$ for $>12 \mathrm{~h}$ at $\sim 100{ }^{\circ} \mathrm{C}$ and, finally, diluting the sample solution with $2 \%$ $\mathrm{HNO}_{3}$. An internal standard solution of $5 \mathrm{ppb} \mathrm{Rh}$ and $\mathrm{Bi}$ was added and the spiked solution was diluted with $2 \% \mathrm{HNO}_{3}$ to a sample/solution weight ratio of $1: 2000$. The internal standard solution was used for monitoring the signal shift during inductively coupled plasma-mass spectrometry (ICP-MS) measurements using an Agilent 7500 s spectrometer at the National Taiwan University, which shows a good stability range with $\sim 10 \%$ variation. The precision was generally better than $\pm 5 \%(2 \sigma)$ for most trace elements, as shown by the statistics on duplicate analyses of five rock standards AGV-1, AGV2, GSP-1, JB-1 and JG-1 (Yang et al., 2005).

\section{2. $\mathrm{Sr}$ and $\mathrm{Nd}$ isotopes}

Samples for isotopic analysis were dissolved in Teflon bombs after being spiked with ${ }^{84} \mathrm{Sr},{ }^{87} \mathrm{Rb},{ }^{150} \mathrm{Nd}$ and ${ }^{147} \mathrm{Sm}$ tracers prior to $\mathrm{HF}+\mathrm{HNO}_{3}$ (with a ratio of $2: 1)$ dissolution. Rubidium, Sr, Sm and Nd were separated using conventional ion exchange procedures and measured using a Finnigan MAT 262 multi-collector mass spectrometer at the Institute of Geology and Geophysics, Chinese Academy of Sciences, China (Yang et al., 2004b). Procedural blanks were $<100$ pg for Sm and $\mathrm{Nd}$ and $<500 \mathrm{pg}$ for $\mathrm{Rb}$ and $\mathrm{Sr} .{ }^{143} \mathrm{Nd} /{ }^{144} \mathrm{Nd}$ were corrected for mass fractionation by normalization to ${ }^{146} \mathrm{Nd} /{ }^{144} \mathrm{Nd}=0.7219$, and ${ }^{87} \mathrm{Sr} /{ }^{86} \mathrm{Sr}$ ratios were normalized to ${ }^{86} \mathrm{Sr} /{ }^{88} \mathrm{Sr}=0.1194$. Typical within-run precision $(2 \sigma)$ for $\mathrm{Sr}$ and $\mathrm{Nd}$ was estimated to be \pm 0.000015 . The measured values for the La Jolla and BCR-1 Nd standards and the NBS-607 $\mathrm{Sr}$ standard were ${ }^{143} \mathrm{Nd} /{ }^{144} \mathrm{Nd}=0.511853 \pm 7\left(2 \sigma_{n}, n=3\right)$ and $0.512604 \pm 7\left(2 \sigma_{n}, n=3\right)$, respectively, and ${ }^{87} \mathrm{Sr} /$ ${ }^{86} \mathrm{Sr}=1.20042 \pm 2\left(2 \sigma_{n}, n=12\right)$ during the period of data acquisition.

\subsection{Zircon Hf isotopes}

Cathodoluminescence (CL) images were obtained using a CAMECA SX50 microprobe at the Institute 
Table 1

Whole rock compositions of the Qianshan pluton granites and mafic microgranular enclaves

\begin{tabular}{|c|c|c|c|c|c|c|c|c|c|c|c|}
\hline \multirow{2}{*}{$\frac{\text { Sample no. }}{\text { Rock type }}$} & JH-07 & JH-08 & JH-09 & JH-12 & JH-15-1 & JH-17-1 & FW01-162 & JH-26 & JH-27 & !JH-28 & JH-29 \\
\hline & \multicolumn{8}{|c|}{ Biotite granites } & \multicolumn{3}{|c|}{ Hornblende granites } \\
\hline \multicolumn{12}{|c|}{ Major elements (wt.\%) } \\
\hline $\mathrm{SiO}_{2}$ & 71.11 & 71.11 & 70.84 & 71.33 & 71.16 & 70.49 & 71.47 & 70.28 & 69.67 & 68.90 & 69.10 \\
\hline $\mathrm{TiO}_{2}$ & 0.25 & 0.25 & 0.23 & 0.27 & 0.27 & 0.25 & 0.23 & 0.32 & 0.18 & 0.18 & 0.18 \\
\hline $\mathrm{Al}_{2} \mathrm{O}_{3}$ & 14.90 & 14.85 & 15.34 & 14.65 & 15.09 & 15.05 & 14.51 & 14.41 & 15.32 & 15.74 & 15.68 \\
\hline $\mathrm{Fe}_{2} \mathrm{O}_{3}$ & 2.30 & 2.29 & 1.99 & 2.59 & 2.34 & 2.21 & 2.40 & 2.89 & 1.98 & 2.01 & 2.01 \\
\hline $\mathrm{MnO}$ & 0.06 & 0.06 & 0.05 & 0.06 & 0.06 & 0.06 & 0.06 & 0.07 & 0.05 & 0.05 & 0.05 \\
\hline $\mathrm{MgO}$ & 0.12 & 0.12 & 0.09 & 0.15 & 0.13 & 0.13 & 0.21 & 0.17 & 0.01 & 0.01 & 0.01 \\
\hline $\mathrm{CaO}$ & 0.99 & 0.97 & 0.93 & 1.04 & 1.07 & 1.03 & 1.17 & 1.08 & 0.69 & 0.72 & 0.72 \\
\hline $\mathrm{Na}_{2} \mathrm{O}$ & 4.30 & 4.12 & 4.41 & 4.23 & 4.24 & 4.22 & 4.57 & 4.14 & 4.53 & 4.64 & 4.67 \\
\hline $\mathrm{K}_{2} \mathrm{O}$ & 5.15 & 5.39 & 5.46 & 5.14 & 5.50 & 5.50 & 5.28 & 5.04 & 6.13 & 6.44 & 6.33 \\
\hline $\mathrm{P}_{2} \mathrm{O}_{5}$ & 0.05 & 0.05 & 0.04 & 0.05 & 0.06 & 0.05 & 0.05 & 0.06 & 0.02 & 0.02 & 0.01 \\
\hline Total & 99.24 & 99.20 & 99.38 & 99.52 & 99.92 & 98.99 & 99.95 & 98.45 & 98.56 & 98.71 & 98.75 \\
\hline \multicolumn{12}{|c|}{ Trace elements (ppm) } \\
\hline $\mathrm{Cr}$ & 4.79 & 3.69 & 6.17 & 5.38 & 3.83 & 4.95 & 2.43 & 3.57 & 3.33 & 4.49 & 2.19 \\
\hline $\mathrm{Ni}$ & 6.62 & 3.25 & 4.02 & 3.15 & 2.73 & 3.43 & 1.82 & 2.48 & 4.22 & 4.83 & 1.52 \\
\hline $\mathrm{Ga}$ & 26.8 & 26.4 & 27.2 & 27.0 & 26.5 & 26.3 & 21.6 & 26.7 & 25.8 & 27.2 & 25.9 \\
\hline $\mathrm{Rb}$ & 191 & 199 & 203 & 202 & 211 & 205 & 197 & 202 & 141 & 140 & 137 \\
\hline $\mathrm{Sr}$ & 88.1 & 89.8 & 94.1 & 91.9 & 93.9 & 94.5 & 82.0 & 101 & 20.3 & 27.5 & 26.9 \\
\hline $\mathrm{Y}$ & 40.3 & 40.8 & 35.9 & 43.4 & 45.3 & 40.3 & 35.6 & 48.3 & 25.0 & 26.2 & 22.1 \\
\hline $\mathrm{Zr}$ & 3951 & 3861 & 345 & 4035 & 1029 & 371 & 319 & 2912 & 5204 & 1462 & 537 \\
\hline $\mathrm{Nb}$ & 27.6 & 26.7 & 24.2 & 29.3 & 28.5 & 26.9 & 26.3 & 33.4 & 20.6 & 19.7 & 20.1 \\
\hline Cs & 2.0 & 2.1 & 2.1 & 2.5 & 2.6 & 2.4 & 2.77 & 3.0 & 1.7 & 1.7 & 1.6 \\
\hline $\mathrm{Ba}$ & 403 & 419 & 434 & 422 & 474 & 503 & 419 & 505 & 108 & 138 & 132 \\
\hline $\mathrm{La}$ & 73.9 & 89.7 & 78.0 & 98.9 & 87.1 & 68.9 & 78.2 & 124 & 91.3 & 159 & 95.7 \\
\hline $\mathrm{Ce}$ & 145 & 174 & 150 & 190 & 172 & 136 & 164 & 235 & 173.1 & 266 & 181 \\
\hline $\operatorname{Pr}$ & 15.1 & 17.7 & 15.3 & 19.0 & 17.8 & 14.2 & 16.9 & 23.2 & 17.4 & 28.3 & 18.2 \\
\hline $\mathrm{Nd}$ & 55.2 & 63.4 & 54.7 & 67.5 & 64.9 & 52.9 & 62.9 & 81.7 & 63.3 & 98.0 & 65.7 \\
\hline $\mathrm{Sm}$ & 9.81 & 10.7 & 9.36 & 11.3 & 11.2 & 9.58 & 10.1 & 13.0 & 9.62 & 12.8 & 9.49 \\
\hline $\mathrm{Eu}$ & 0.69 & 0.72 & 0.74 & 0.73 & 0.74 & 0.72 & 0.74 & 0.73 & 0.15 & 0.18 & 0.17 \\
\hline $\mathrm{Gd}$ & 8.24 & 8.90 & 7.75 & 9.34 & 9.23 & 7.99 & 9.04 & 10.67 & 7.54 & 9.90 & 7.40 \\
\hline $\mathrm{Tb}$ & 1.35 & 1.40 & 1.23 & 1.48 & 1.49 & 1.32 & 1.22 & 1.64 & 1.01 & 1.21 & 0.96 \\
\hline Dy & 7.10 & 7.25 & 6.37 & 7.71 & 7.81 & 6.93 & 6.54 & 8.42 & 4.80 & 5.30 & 4.41 \\
\hline Ho & 1.35 & 1.38 & 1.21 & 1.46 & 1.46 & 1.32 & 1.27 & 1.60 & 0.88 & 0.96 & 0.81 \\
\hline $\mathrm{Er}$ & 3.94 & 3.98 & 3.51 & 4.24 & 4.26 & 3.79 & 3.37 & 4.67 & 2.58 & 2.75 & 2.37 \\
\hline $\mathrm{Tm}$ & 0.61 & 0.62 & 0.54 & 0.65 & 0.65 & 0.57 & 0.51 & 0.72 & 0.40 & 0.40 & 0.36 \\
\hline $\mathrm{Yb}$ & 3.83 & 3.85 & 3.41 & 4.10 & 4.01 & 3.60 & 3.47 & 4.49 & 2.63 & 2.61 & 2.45 \\
\hline $\mathrm{Lu}$ & 0.57 & 0.59 & 0.51 & 0.61 & 0.60 & 0.53 & 0.55 & 0.68 & 0.44 & 0.43 & 0.40 \\
\hline Hf & 10.2 & 9.9 & 8.4 & 10.4 & 9.2 & 8.8 & 8.6 & 11.4 & 11.6 & 11.3 & 11.3 \\
\hline $\mathrm{Ta}$ & 2.15 & 2.14 & 1.97 & 2.27 & 2.25 & 1.99 & 1.83 & 2.57 & 1.17 & 1.06 & 1.03 \\
\hline $\mathrm{Pb}$ & 33.0 & 32.5 & 37.1 & 39.2 & 33.6 & 27.7 & 31.3 & 33.2 & 27.4 & 27.5 & 62.7 \\
\hline Th & 17.8 & 21.3 & 18.1 & 21.4 & 19.5 & 16.4 & 21.4 & 28.3 & 16.8 & 19.1 & 15.3 \\
\hline $\mathrm{U}$ & 5.25 & 5.21 & 5.08 & 6.02 & 3.96 & 3.26 & 5.24 & 6.97 & 2.86 & 2.57 & 2.67 \\
\hline
\end{tabular}

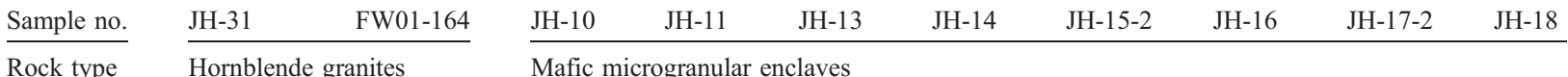

Major elements (wt.\%)

$\mathrm{SiO}_{2} \quad 66.75 \quad 71.74$

$\mathrm{TiO}_{2}$

0.3

$\begin{array}{lll}57.52 & 58.89 & 60.16\end{array}$

55.96

62.60

$60.20 \quad 61.42$

61.69

$\mathrm{Al}_{2} \mathrm{O}_{3}$

15.69

14.17

$\begin{array}{rr}1.16 & 1.02 \\ 16.34 & 15.80\end{array}$

0.96

1.19

0.59

1.07

0.78

0.63

$\mathrm{Fe}_{2} \mathrm{O}_{3}$

$3.63 \quad 2.67$

$\begin{array}{rr}16.34 & 15.80 \\ 8.91 & 8.97\end{array}$

16.62

16.95

14.45

16.04

15.78

14.30

$\mathrm{MnO}$

$0.07 \quad 0.05$

0.23

0.25

0.18

8.68
0.20

6.89

6.19

7.04

$\mathrm{MgO}$

$\begin{array}{ll}0.01 & 0.04 \\ 1.07 & 1.01\end{array}$

1.79

1.38

1.08

2.71

0.22

0.17

0.20

$\mathrm{CaO}$

3.87

3.71

2.75

4.36

2.28

$\begin{array}{ll}1.69 & 2.75\end{array}$

3.26

$\begin{array}{lll}3.52 & 4.08 & 3.43\end{array}$


Table 1 (continued)

\begin{tabular}{|c|c|c|c|c|c|c|c|c|c|c|}
\hline \multirow{2}{*}{$\frac{\text { Sample no. }}{\text { Rock type }}$} & JH-31 & FW01-164 & JH-10 & JH-11 & JH-13 & $\mathrm{JH}-14$ & JH-15-2 & JH-16 & JH-17-2 & JH-18 \\
\hline & \multicolumn{2}{|c|}{ Hornblende granites } & \multicolumn{8}{|c|}{ Mafic microgranular enclaves } \\
\hline \multicolumn{11}{|c|}{ Major elements (wt.\%) } \\
\hline $\mathrm{Na}_{2} \mathrm{O}$ & 4.70 & 4.44 & 5.41 & 5.27 & 5.39 & 5.76 & 5.27 & 5.95 & 6.25 & 5.64 \\
\hline $\mathrm{K}_{2} \mathrm{O}$ & 6.32 & 5.74 & 2.26 & 2.67 & 3.07 & 2.16 & 2.91 & 2.54 & 1.45 & 2.03 \\
\hline $\mathrm{P}_{2} \mathrm{O}_{5}$ & 0.02 & 0.02 & 0.43 & 0.38 & 0.33 & 0.40 & 0.15 & 0.34 & 0.18 & 0.21 \\
\hline Total & 98.58 & 100.09 & 97.92 & 98.34 & 98.09 & 98.37 & 98.70 & 98.39 & 99.05 & 98.43 \\
\hline \multicolumn{11}{|c|}{ Trace elements (ppm) } \\
\hline $\mathrm{Cr}$ & 2.68 & 3.00 & 4.55 & 3.60 & 2.88 & 5.16 & 65.3 & 7.09 & 52.2 & 88.5 \\
\hline $\mathrm{Ni}$ & 2.66 & 3.23 & 7.44 & 5.63 & 3.52 & 11.1 & 29.7 & 8.64 & 29.0 & 45.3 \\
\hline $\mathrm{Ga}$ & 29.4 & 18.8 & 30.9 & 31.9 & 32.0 & 25.9 & 26.7 & 24.7 & 24.8 & 23.1 \\
\hline $\mathrm{Rb}$ & 138 & 123 & 184 & 198 & 222 & 180 & 191 & 145 & 82 & 146 \\
\hline $\mathrm{Sr}$ & 25.8 & 15.9 & 254 & 170 & 336 & 289 & 87.1 & 250 & 175 & 102 \\
\hline $\mathrm{Y}$ & 42.3 & 27.4 & 77.3 & 92.1 & 55.3 & 45.4 & 61.8 & 42.9 & 30.8 & 45.9 \\
\hline $\mathrm{Zr}$ & 10,270 & 671 & 191 & 334 & 259 & 281 & 260 & 337 & 226 & 255 \\
\hline $\mathrm{Nb}$ & 28.9 & 23.6 & 56.5 & 62.0 & 55.9 & 23.0 & 30.5 & 20.3 & 16.8 & 22.3 \\
\hline Cs & 2.1 & 1.91 & 2.6 & 4.1 & 3.9 & 2.2 & 2.3 & 2.3 & 1.0 & 1.8 \\
\hline $\mathrm{Ba}$ & 119 & 45.4 & 239 & 277 & 455 & 282 & 270 & 532 & 241 & 249 \\
\hline $\mathrm{La}$ & 268 & 127 & 70.0 & 60.5 & 41.5 & 70.8 & 53.1 & 70.8 & 56.9 & 58.5 \\
\hline $\mathrm{Ce}$ & 484 & 249 & 180 & 164 & 101 & 153 & 133 & 145 & 118 & 132 \\
\hline $\operatorname{Pr}$ & 51.4 & 22.7 & 22.8 & 21.6 & 12.7 & 17.0 & 16.7 & 15.5 & 12.8 & 15.4 \\
\hline $\mathrm{Nd}$ & 175 & 82.1 & 96.0 & 93.3 & 54.0 & 65.5 & 69.3 & 58.8 & 47.3 & 59.8 \\
\hline $\mathrm{Sm}$ & 22.5 & 11.9 & 19.8 & 20.2 & 11.5 & 11.7 & 14.3 & 10.1 & 8.20 & 10.8 \\
\hline $\mathrm{Eu}$ & 0.16 & 0.16 & 0.91 & 0.92 & 1.04 & 1.08 & 0.68 & 1.27 & 0.86 & 0.73 \\
\hline $\mathrm{Gd}$ & 17.2 & 10.8 & 16.1 & 16.5 & 9.74 & 9.52 & 11.6 & 8.39 & 6.67 & 8.88 \\
\hline $\mathrm{Tb}$ & 2.02 & 1.27 & 2.72 & 2.93 & 1.71 & 1.52 & 2.00 & 1.34 & 1.05 & 1.45 \\
\hline Dy & 8.59 & 6.33 & 14.4 & 16.0 & 9.31 & 7.89 & 10.8 & 7.12 & 5.39 & 7.71 \\
\hline Но & 1.55 & 1.12 & 2.67 & 3.01 & 1.79 & 1.47 & 2.01 & 1.37 & 1.01 & 1.47 \\
\hline $\mathrm{Er}$ & 4.53 & 2.89 & 7.44 & 8.60 & 5.20 & 4.25 & 5.69 & 4.08 & 2.89 & 4.22 \\
\hline $\mathrm{Tm}$ & 0.67 & 0.40 & 1.08 & 1.32 & 0.81 & 0.65 & 0.86 & 0.63 & 0.44 & 0.64 \\
\hline $\mathrm{Yb}$ & 4.43 & 2.73 & 6.47 & 8.06 & 4.98 & 3.95 & 5.17 & 3.92 & 2.64 & 3.83 \\
\hline $\mathrm{Lu}$ & 0.73 & 0.44 & 0.93 & 1.18 & 0.74 & 0.57 & 0.75 & 0.58 & 0.39 & 0.55 \\
\hline Hf & 22.9 & 14.7 & 5.2 & 8.3 & 6.5 & 6.3 & 6.4 & 7.5 & 5.5 & 6.6 \\
\hline $\mathrm{Ta}$ & 1.44 & 1.17 & 2.58 & 3.22 & 2.86 & 1.43 & 1.60 & 1.45 & 1.19 & 1.52 \\
\hline $\mathrm{Pb}$ & 27.9 & 25.33 & 33.7 & 32.7 & 27.4 & 26.4 & 35.5 & 29.2 & 15.9 & 17.3 \\
\hline Th & 31.4 & 16.7 & 11.8 & 12.9 & 12.7 & 12.5 & 12.0 & 14.6 & 11.3 & 13.8 \\
\hline $\mathrm{U}$ & 4.57 & 3.17 & 6.82 & 7.07 & 6.68 & 5.02 & 4.15 & 12.1 & 2.84 & 5.57 \\
\hline
\end{tabular}

\begin{tabular}{|c|c|c|c|c|c|c|c|c|}
\hline Sample no. & JH-19 & JH-20 & JH-21 & JH-22 & JH-23 & JH-24 & JH-25 & JH-30 \\
\hline Rock type & \multicolumn{8}{|c|}{ Mafic microgranular enclave } \\
\hline \multicolumn{9}{|c|}{ Major elements (wt.\%) } \\
\hline $\mathrm{SiO}_{2}$ & 61.03 & 60.39 & 67.04 & 59.88 & 60.24 & 60.87 & 62.89 & 60.04 \\
\hline $\mathrm{TiO}_{2}$ & 0.96 & 0.82 & 0.47 & 0.83 & 0.98 & 0.96 & 0.46 & 0.82 \\
\hline $\mathrm{Al}_{2} \mathrm{O}_{3}$ & 17.08 & 15.79 & 15.20 & 15.77 & 16.30 & 15.01 & 16.68 & 15.20 \\
\hline $\mathrm{Fe}_{2} \mathrm{O}_{3}$ & 6.09 & 5.38 & 3.60 & 4.96 & 7.29 & 6.60 & 5.06 & 6.42 \\
\hline $\mathrm{MnO}$ & 0.15 & 0.10 & 0.10 & 0.07 & 0.20 & 0.17 & 0.13 & 0.15 \\
\hline $\mathrm{MgO}$ & 1.67 & 2.81 & 0.55 & 2.91 & 1.31 & 2.27 & 0.62 & 0.91 \\
\hline $\mathrm{CaO}$ & 3.39 & 4.16 & 1.80 & 4.40 & 2.99 & 3.84 & 2.01 & 4.09 \\
\hline $\mathrm{Na}_{2} \mathrm{O}$ & 5.67 & 5.07 & 4.50 & 4.61 & 5.40 & 5.41 & 5.24 & 4.34 \\
\hline $\mathrm{K}_{2} \mathrm{O}$ & 2.15 & 3.61 & 5.07 & 4.34 & 3.04 & 2.84 & 4.78 & 6.08 \\
\hline $\mathrm{P}_{2} \mathrm{O}_{5}$ & 0.32 & 0.27 & 0.15 & 0.27 & 0.37 & 0.26 & 0.16 & 0.27 \\
\hline Total & 98.50 & 98.40 & 98.48 & 98.03 & 98.10 & 98.22 & 98.03 & 98.31 \\
\hline \multicolumn{9}{|c|}{ Trace elements (ppm) } \\
\hline $\mathrm{Cr}$ & 3.62 & 64.5 & 13.5 & 66.4 & 3.94 & 41.0 & 3.13 & 7.43 \\
\hline $\mathrm{Ni}$ & 10.1 & 37.1 & 9.46 & 36.1 & 3.95 & 22.9 & 3.67 & 6.41 \\
\hline $\mathrm{Ga}$ & 32.3 & 22.5 & 25.4 & 21.6 & 28.2 & 23.7 & 25.2 & 22.1 \\
\hline
\end{tabular}


Table 1 (continued)

\begin{tabular}{|c|c|c|c|c|c|c|c|c|}
\hline \multirow{2}{*}{$\frac{\text { Sample no. }}{\text { Rock type }}$} & JH-19 & JH-20 & JH-21 & $\mathrm{JH}-22$ & $\mathrm{JH}-23$ & JH-24 & JH-25 & JH-30 \\
\hline & \multicolumn{8}{|c|}{ Mafic microgranular enclave } \\
\hline \multicolumn{9}{|c|}{ Trace elements (ppm) } \\
\hline $\mathrm{Rb}$ & 190 & 118 & 181 & 113 & 199 & 122 & 226 & 134 \\
\hline $\mathrm{Sr}$ & 477 & 639 & 171 & 835 & 224 & 176 & 151 & 122 \\
\hline Y & 53.0 & 36.1 & 51.9 & 35.7 & 60.3 & 43.9 & 43.2 & 37.8 \\
\hline $\mathrm{Zr}$ & 340 & 212 & 318 & 243 & 189 & 285 & 480 & 379 \\
\hline $\mathrm{Nb}$ & 35.7 & 14.9 & 41.3 & 11.3 & 46.9 & 23.9 & 32.2 & 18.9 \\
\hline $\mathrm{Cs}$ & 2.6 & 1.0 & 2.0 & 0.5 & 3.8 & 1.1 & 4.4 & 1.2 \\
\hline $\mathrm{Ba}$ & 606 & 1115 & 729 & 1426 & 403 & 552 & 555 & 1206 \\
\hline $\mathrm{La}$ & 69.8 & 52.1 & 39.1 & 50.2 & 47.3 & 65.5 & 54.7 & 44.5 \\
\hline $\mathrm{Ce}$ & 153 & 105 & 97.1 & 94.8 & 118 & 142 & 117 & 102 \\
\hline $\operatorname{Pr}$ & 17.2 & 11.3 & 12.3 & 9.9 & 14.9 & 15.7 & 13.0 & 12.2 \\
\hline $\mathrm{Nd}$ & 66.4 & 42.7 & 51.6 & 37.3 & 62.9 & 60.2 & 48.8 & 50.9 \\
\hline $\mathrm{Sm}$ & 11.6 & 7.28 & 10.6 & 6.11 & 13.0 & 10.8 & 8.64 & 9.91 \\
\hline $\mathrm{Eu}$ & 1.14 & 1.20 & 0.85 & 1.37 & 0.80 & 1.07 & 0.84 & 1.17 \\
\hline $\mathrm{Gd}$ & 9.65 & 6.08 & 8.90 & 5.22 & 10.6 & 8.95 & 7.24 & 8.51 \\
\hline $\mathrm{Tb}$ & 1.60 & 0.97 & 1.59 & 0.82 & 1.85 & 1.44 & 1.22 & 1.38 \\
\hline Dy & 8.58 & 5.38 & 8.77 & 4.67 & 10.1 & 7.57 & 6.56 & 7.08 \\
\hline Ho & 1.68 & 1.11 & 1.67 & 1.01 & 1.91 & 1.43 & 1.29 & 1.30 \\
\hline $\mathrm{Er}$ & 5.06 & 3.39 & 4.80 & 3.19 & 5.48 & 4.06 & 3.93 & 3.63 \\
\hline $\mathrm{Tm}$ & 0.79 & 0.52 & 0.72 & 0.47 & 0.84 & 0.61 & 0.64 & 0.54 \\
\hline $\mathrm{Yb}$ & 4.99 & 3.10 & 4.44 & 2.72 & 5.13 & 3.75 & 4.20 & 3.35 \\
\hline $\mathrm{Lu}$ & 0.74 & 0.46 & 0.65 & 0.41 & 0.75 & 0.55 & 0.64 & 0.52 \\
\hline $\mathrm{Hf}$ & 8.5 & 5.2 & 7.2 & 5.8 & 4.8 & 6.7 & 9.8 & 7.3 \\
\hline $\mathrm{Ta}$ & 2.72 & 1.13 & 2.13 & 0.93 & 2.54 & 1.76 & 2.19 & 1.24 \\
\hline $\mathrm{Pb}$ & 22.7 & 23.8 & 28.7 & 24.4 & 17.4 & 21.6 & 26.7 & 27.8 \\
\hline Th & 17.0 & 15.1 & 10.5 & 16.7 & 12.1 & 14.3 & 17.9 & 8.9 \\
\hline $\mathrm{U}$ & 11.9 & 73.7 & 10.4 & 127 & 6.98 & 9.70 & 7.93 & 2.28 \\
\hline
\end{tabular}

of Geology and Geophysics, Chinese Academy of Sciences in Beijing, in order to identify zircon internal textures and choose potential target sites for Hf analyses. The working conditions during the CL imaging were at $15 \mathrm{kV}$.

In situ zircon $\mathrm{Hf}$ isotopic analyses were carried out at the Institute of Geology and Geophysics, Chinese Academy of Sciences using a Neptune MC-ICPMS with an ArF excimer laser ablation system. During analyses, the spot sizes of 32 and $63 \mu \mathrm{m}$ and a laser repetition rate of 10 $\mathrm{Hz}$ with $100 \mathrm{~mJ}$ were used. Details of the technique are described by $\mathrm{Xu}$ et al. (2004). During analyses, the ${ }^{176} \mathrm{Hf} /{ }^{177} \mathrm{Hf}$ and ${ }^{176} \mathrm{Lu} /{ }^{177} \mathrm{Hf}$ ratios of standard zircon (91500) were $0.282294 \pm 15\left(2 \sigma_{n}, n=20\right)$ and 0.00031 , similar to the commonly accepted ${ }^{176} \mathrm{Hf} /{ }^{177} \mathrm{Hf}$ ratio of $0.282284 \pm 3(1 \sigma)$ measured using the solution method (Goolaerts et al., 2004; Woodhead et al., 2004).

The notations of $\varepsilon_{\mathrm{Hf}}, f_{\mathrm{Lu} / \mathrm{Hf}}$ and $T_{\mathrm{Hf}}$ are defined as:

$$
\begin{aligned}
\varepsilon_{\mathrm{Hf}}(0)= & \left(\left({ }^{176} \mathrm{Hf} /{ }^{177} \mathrm{Hf}\right)_{\mathrm{S}} /\left({ }^{176} \mathrm{Hf} /{ }^{177} \mathrm{Hf}\right)_{\mathrm{CHUR}, 0}-1\right) \times \\
& 10,000 \\
\varepsilon_{\mathrm{Hf}}(t)= & \left(\left({ }^{176} \mathrm{Hf} /{ }^{177} \mathrm{Hf}\right)_{\mathrm{S}}-\left({ }^{176} \mathrm{Lu} /{ }^{177} \mathrm{Hf}\right)_{\mathrm{S}} \times\left(e^{\lambda t}-1\right)\right) / \\
& \left(\left({ }^{176} \mathrm{Hf} /{ }^{177} \mathrm{Hf}\right)_{\mathrm{CHUR}, 0}-\left({ }^{176} \mathrm{Lu} /{ }^{177} \mathrm{Hf}\right)_{\mathrm{CHUR}} \times\right. \\
& \left.\left.\left(e^{\lambda t}-1\right)\right)-1\right) \times 10,000
\end{aligned}
$$

$\begin{aligned} T_{\mathrm{DM}}= & 1 / \lambda \times\left(1+\left(\left({ }^{176} \mathrm{Hf} /{ }^{177} \mathrm{Hf}\right)_{\mathrm{S}}-\left({ }^{176} \mathrm{Hf} /{ }^{177} \mathrm{Hf}\right)_{\mathrm{DM}}\right) /\right. \\ & \left.\left(\left({ }^{176} \mathrm{Lu} /{ }^{177} \mathrm{Hf}\right)_{\mathrm{S}}-\left({ }^{176} \mathrm{Lu} /{ }^{177} \mathrm{Hf}\right)_{\mathrm{DM}}\right)\right) \\ f_{\mathrm{Lu} / \mathrm{Hf}}= & \left({ }^{176} \mathrm{Lu} /{ }^{177} \mathrm{Hf}\right)_{\mathrm{S}} /\left({ }^{176} \mathrm{Lu} /{ }^{177} \mathrm{Hf}\right)_{\mathrm{CHUR}}-1\end{aligned}$

where, $\left({ }^{176} \mathrm{Lu} /{ }^{177} \mathrm{Hf}\right)_{\mathrm{S}}$ and $\left({ }^{176} \mathrm{Hf} /{ }^{177} \mathrm{Hf}\right)_{\mathrm{S}}$ are the measured values of samples, $\left({ }^{176} \mathrm{Lu} /{ }^{177} \mathrm{Hf}\right)_{\mathrm{CHUR}}=0.0332$ and $\left({ }^{176} \mathrm{Hf} /{ }^{177} \mathrm{Hf}\right)_{\mathrm{CHUR}, 0}=0.282772 \quad$ (Blichert-Toft and Albarede, 1997); $\left({ }^{176} \mathrm{Lu} /{ }^{177} \mathrm{Hf}\right)_{\mathrm{DM}}=0.0384$ and $\left({ }^{176} \mathrm{Hf} /{ }^{177} \mathrm{Hf}\right)_{\mathrm{DM}}=0.28325$ (Griffin et al., 2000), $t=$ crystallization time of zircon. $\lambda=1.867 \times 10^{-11} \mathrm{yr}^{-1}$ (Soderlund et al., 2004) was used in our calculations.

\section{Results}

\subsection{Major and trace element data}

All granite samples from the Qianshan pluton are high in silica and alkalis, with $\mathrm{SiO}_{2}$ ranging from 66.7 to $71.3 \mathrm{wt} . \%$ and total $\mathrm{K}_{2} \mathrm{O}+\mathrm{Na}_{2} \mathrm{O}$ varying from 9.2 to 11.0 wt.\% (Table 1). They all plot as alkaline rocks on the alkali vs. silica diagram (Fig. 3a). The biotite granites are mostly peraluminous with $\mathrm{A} / \mathrm{CNK}$ (molar ratio of $\mathrm{Al}_{2} \mathrm{O}_{3} /\left[\mathrm{CaO}+\mathrm{Na}_{2} \mathrm{O}+\mathrm{K}_{2} \mathrm{O}\right]$ ) and $\mathrm{A} / \mathrm{NK}$ ratios ranging from 1.01 to 1.03 and 1.10 to 1.18 , respectively, 

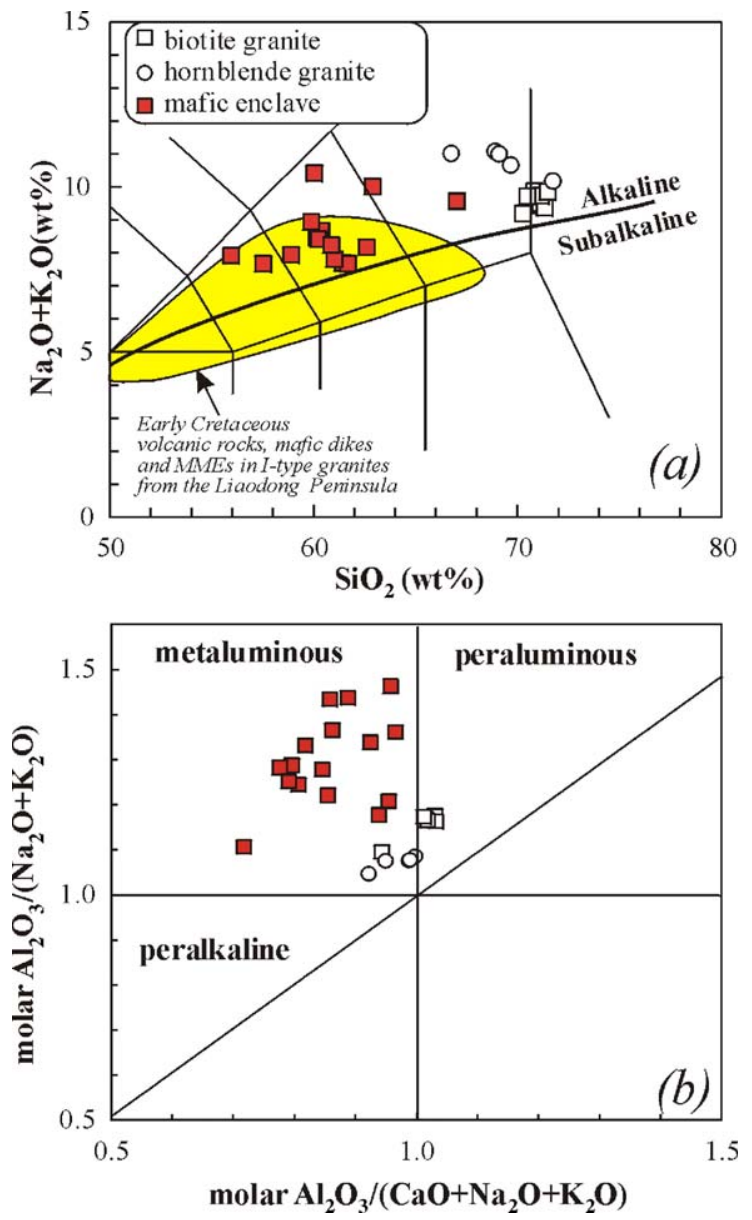

Fig. 3. Plots of (a) $\mathrm{Na}_{2} \mathrm{O}+\mathrm{K}_{2} \mathrm{O}$ vs. $\mathrm{SiO}_{2}$ and (b) $\mathrm{A} / \mathrm{NK}\left[\mathrm{Al}_{2} \mathrm{O}_{3} /\right.$ $\left.\left(\mathrm{Na}_{2} \mathrm{O}+\mathrm{K}_{2} \mathrm{O}\right)\right]$ vs. A/CNK [molar ratio $\mathrm{Al}_{2} \mathrm{O}_{3} /\left(\mathrm{CaO}+\mathrm{Na}_{2} \mathrm{O}+\mathrm{K}_{2} \mathrm{O}\right)$ ] for mafic enclaves and host granitoids from the Qianshan pluton in the Liaodong Peninsula, northeastern China. The field of Early Cretaceous volcanic rocks, MMEs in I-type granites and mafic dykes in (a) is from Yang et al. (2004b).

although one sample (FW01-162) is metaluminous (Fig. 3b). All hornblende granites are metaluminous, with $\mathrm{A} / \mathrm{CNK}$ and $\mathrm{A} / \mathrm{NK}$ ratios ranging from 0.92 to 1.0 and 1.05 to 1.09 , respectively (Fig. 3b). They have lower total $\mathrm{Fe}_{2} \mathrm{O}_{3}$ (2.0-3.6 wt.\%), $\mathrm{MnO}(0.05-0.07$ wt.\%), $\mathrm{CaO}$ (0.7-1.1 wt.\%), $\mathrm{TiO}_{2}(0.18-0.33$ wt.\%) and $\mathrm{P}_{2} \mathrm{O}_{5}(0.01-0.06$ wt.\%) contents (Table 1) and $\mathrm{Yb} / \mathrm{Hf}$ ratios, but higher $\mathrm{Zr}, \mathrm{Nb}, \mathrm{Ce}$ and $\mathrm{Y}$ concentrations and $\left(\mathrm{K}_{2} \mathrm{O}+\mathrm{Na}_{2} \mathrm{O}\right) / \mathrm{CaO}, \mathrm{Ga} / \mathrm{Al}_{2} \mathrm{O}_{3}, \mathrm{Fe}_{2} \mathrm{O}_{3 \mathrm{~T}} /$ $\mathrm{MgO}$ and $\mathrm{Rb} / \mathrm{Sr}$ ratios than the mafic enclaves (Fig. 4).

Chondrite-normalized REE patterns of the hornblende and biotite granites (Fig. 6a and b) invariably show relative enrichment of light rare earth elements (LREEs), with high $(\mathrm{La} / \mathrm{Yb})_{\mathrm{N}}$ ratios (14-44) and significant negative Eu anomalies. In the PM-normal- ized spidergrams (Fig. 7a and b), all the granites show negative anomalies in $\mathrm{Ba}, \mathrm{Nb}, \mathrm{Ta}, \mathrm{Sr}, \mathrm{P}, \mathrm{Eu}$ and $\mathrm{Ti}$, features characteristic of A-type granites. They are enriched in $\mathrm{Rb}, \mathrm{U}$, Th, LREEs, $\mathrm{Zr}$ and Hf. Significant features to note are the abundance levels of LREE, $\mathrm{Eu}, \mathrm{Sr}$ and $\mathrm{Ba}$ of the biotite granites which are higher than those of the hornblende granites (Fig. 7).

Mafic enclaves have $\mathrm{SiO}_{2}$ contents ranging from 57.5 to 67.0 wt.\% with relatively high Mg-numbers $(\mathrm{Mg} \#=23.4$ to 54.0) (Table 1). They also have higher total $\mathrm{Fe}_{2} \mathrm{O}_{3}, \mathrm{MnO}, \mathrm{CaO}, \mathrm{TiO}_{2}, \mathrm{P}_{2} \mathrm{O}_{5}, \mathrm{Ba}$ and $\mathrm{Sr}$, but lower $\mathrm{K}_{2} \mathrm{O}$ and $\mathrm{Zr}$ contents and $\mathrm{Rb} / \mathrm{Sr}$ and total $\mathrm{Fe}_{2} \mathrm{O}_{3} / \mathrm{MgO}$ ratios than the host granites (Table 1, Figs. 4 and 5). Chondrite-normalized REE patterns for the enclaves are marked by enrichment in the LREEs, variable negative Eu anomalies, and relatively high and variable HREE contents (Fig. 6c). In the primitive mantle-normalized trace element variation diagram (Fig. 7c), most enclave samples show the characteristic negative anomalies in $\mathrm{Ba}, \mathrm{Nb}, \mathrm{Ta}, \mathrm{Sr}$, $\mathrm{P}, \mathrm{Eu}$ and $\mathrm{Ti}$, whereas the samples with high $\mathrm{MgO}$ contents (e.g., JH-22) do not show negative $\mathrm{Ba}$ and $\mathrm{Sr}$ anomalies (Fig. 7c).

\section{2. $\mathrm{Sr}$ and Nd isotope data}

Rubidium, Sr, Sm and Nd concentrations, ${ }^{143} \mathrm{Nd} /$ ${ }^{144} \mathrm{Nd}$ and ${ }^{87} \mathrm{Sr} /{ }^{86} \mathrm{Sr}$ ratios, and $T_{\mathrm{DM}}$ ages for MMEs and granitoids are listed in Table 2 . The initial ${ }^{87} \mathrm{Sr} /{ }^{86} \mathrm{Sr}$ ratios and $\varepsilon_{\mathrm{Nd}}(t)$ values have been calculated at $126 \pm$ $2 \mathrm{Ma}$ on the basis of zircon $\mathrm{U}-\mathrm{Pb}$ dating of rocks from the batholith (Wu et al., 2005a). Depleted mantle model ages $\left(T_{\mathrm{DM}}\right)$ are reported using the model of DePaolo (1981). The data are shown in a plot of $\varepsilon_{\mathrm{Nd}}(t)$ versus $\left({ }^{87} \mathrm{Sr} /{ }^{86} \mathrm{Sr}\right)_{i}$ in Fig. 8, and compared on that diagram with published compositional fields for Late Jurassic and Early Cretaceous granites from the area (Yang et al., 2004b; Wu et al., 2005b).

Two groups of granites can be distinguished on the basis of $\mathrm{Sr}$ and $\mathrm{Nd}$ isotopic compositions (Fig. 8). The hornblende granite has relatively high ${ }^{87} \mathrm{Rb} /{ }^{86} \mathrm{Sr}(16.8-$ $24.2)$ and $\left({ }^{87} \mathrm{Sr} /{ }^{86} \mathrm{Sr}\right)_{i}(0.7258-0.7281)$ ratios and highly negative $\varepsilon_{\mathrm{Nd}}(t)$ values of -15.8 to -16.5 . However, the biotite granite has different $\mathrm{Sr}$ and $\mathrm{Nd}$ isotopic compositions, with low ${ }^{87} \mathrm{Rb} /{ }^{86} \mathrm{Sr}(6.13-6.43)$ and initial ${ }^{87} \mathrm{Sr} /{ }^{86} \mathrm{Sr}(0.7132-0.7135)$ ratios and negative $\varepsilon_{\mathrm{Nd}}(t)$ values of -12.9 to -13.3 . The mafic enclaves have similar $\mathrm{Sr}$ and $\mathrm{Nd}$ isotopic compositions to the biotite granite, with variable ${ }^{87} \mathrm{Rb} /{ }^{86} \mathrm{Sr}(0.39-6.14)$ and initial ${ }^{87} \mathrm{Sr} /{ }^{86} \mathrm{Sr}(0.7097-0.7133)$ ratios and negative $\varepsilon_{\mathrm{Nd}}(t)(-11.9$ to -13.6$)$ values. 

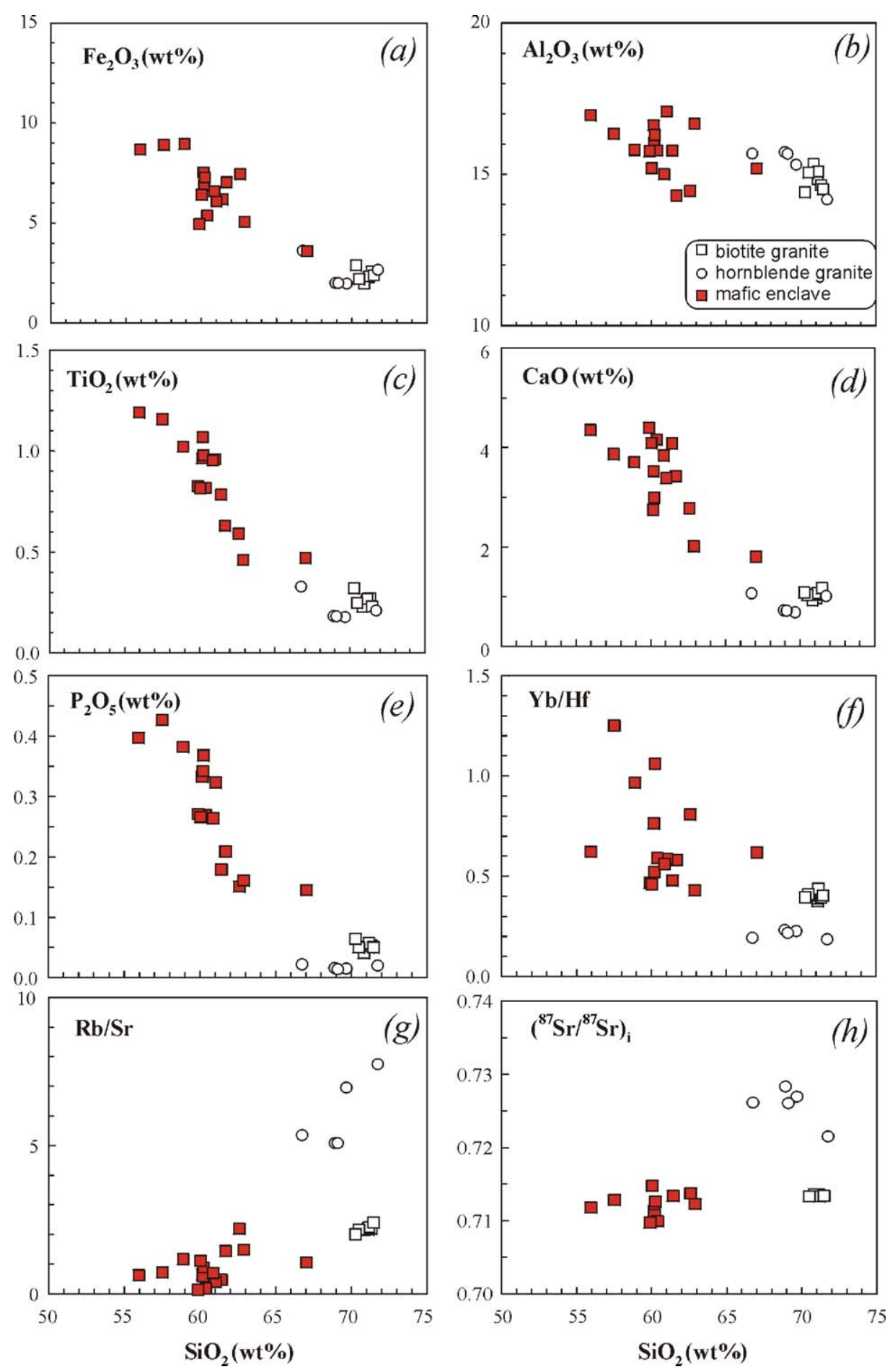

Fig. 4. Various oxide plots [(a), $\mathrm{Fe}_{2} \mathrm{O}_{3}$, (b), $\mathrm{Al}_{2} \mathrm{O}_{3},(\mathrm{c}), \mathrm{TiO}_{2},(\mathrm{~d}), \mathrm{CaO}$ and (e), $\mathrm{P}_{2} \mathrm{O}_{5}$ (all expressed in wt.\%)], trace element plots [(f), $\mathrm{Yb} / \mathrm{Hf}$ and (g), $\mathrm{Rb} / \mathrm{Sr}]$ and isotopic ratios $\left[(\mathrm{h})\left({ }^{87} \mathrm{Sr} /{ }^{86} \mathrm{Sr}\right)_{i}\right]$ vs. $\mathrm{SiO}_{2}$ diagrams for enclaves and host rocks from the Qianshan pluton in the Liaodong Peninsula, northeastern China. 

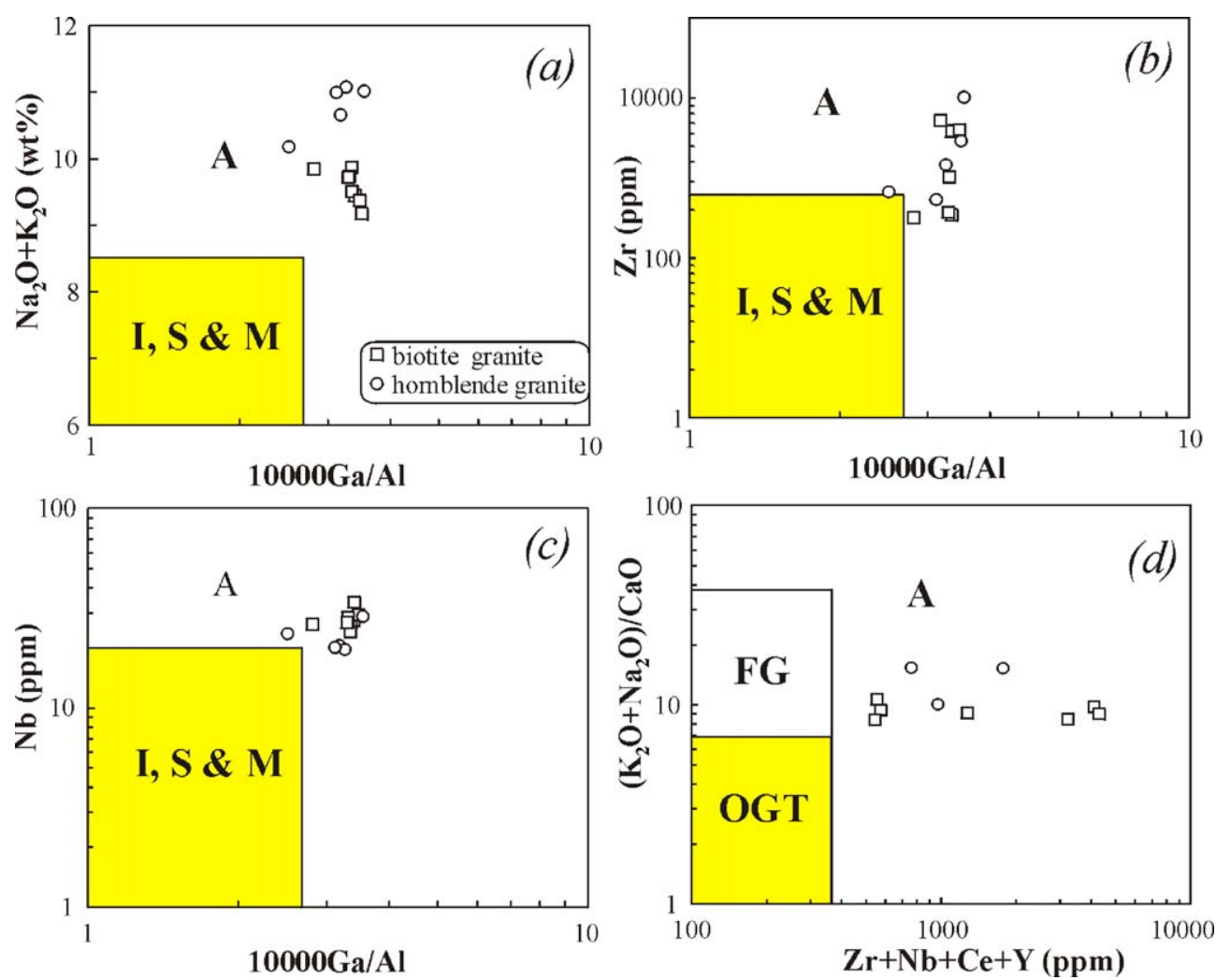

Fig. 5. (a) $\mathrm{Na}_{2} \mathrm{O}+\mathrm{K}_{2} \mathrm{O}$, (b) $\mathrm{Zr}$ and (c) $\mathrm{Nb}$ vs. $10,000 \mathrm{Ga} / \mathrm{Al}$ and (d) $\left(\mathrm{Na}_{2} \mathrm{O}+\mathrm{K}_{2} \mathrm{O}\right) / \mathrm{CaO}$ vs. $(\mathrm{Zr}+\mathrm{Nb}+\mathrm{Ce}+\mathrm{Y})$ discrimination diagrams of Whalen et al. (1987), showing the A-type nature of the Qianshan granites. I, S \& M: unfractionated M-, I- and S-type granites; FG: fractionated felsic granites.

\subsection{Zircon Hf isotope data}

Cathodoluminescence images and in situ Hf isotopic analyses of zircons from the Qianshan granites and mafic enclaves are listed in Supplementary Data file online and shown in Figs. 9 and 10. Zircons from the hornblende granites are fairly homogeneous in CL images (Fig. 9a), but have variable ${ }^{176} \mathrm{Hf} /{ }^{177} \mathrm{Hf}$ ratios $(0.282161$ to 0.282374$)$, with $\varepsilon_{\mathrm{Hf}}(t)$ values ranging from -11.5 to -18.9 (Fig. 10a). Zircons from biotite granites have cores and rims and show strong oscillatory zoning (Fig. 9b). They have a large range of ${ }^{176} \mathrm{Hf} /{ }^{177} \mathrm{Hf}$ ratios, from 0.282321 to 0.282524 , with $\varepsilon_{\mathrm{Hf}}(t)$ values ranging from -6.3 to -16.4 (Fig. 10b). The cores have higher ${ }^{176} \mathrm{Hf} /{ }^{177} \mathrm{Hf}$ ratios than the zircon rims (Fig. 9b). Zircons from MMEs show complex patterns in CL images or are characterized by irregular crystal forms indicative of corrosion (Fig. 9c and d). Zircons from enclaves also have variable ${ }^{176} \mathrm{Hf} /{ }^{177} \mathrm{Hf}$ ratios $(0.282213$ to 0.282513$)$ with $\varepsilon_{\mathrm{Hf}}(t)$ ranging from -6.9 to -17.1 (Fig. 10c). It is evident that the zircons in MMEs have high ${ }^{176} \mathrm{Yb} /{ }^{177} \mathrm{Hf}$ ratios and $\varepsilon_{\mathrm{Hf}}(t)$ values, whereas those in the hornblende granites have low ${ }^{176} \mathrm{Yb} /{ }^{177} \mathrm{Hf}$ ratios and $\varepsilon_{\mathrm{Hf}}(t)$ values.
Zircons in the biotite granites have intermediate ${ }^{176} \mathrm{Yb} /{ }^{177} \mathrm{Hf}$ ratios and $\varepsilon_{\mathrm{Hf}}(t)$ values between zircons in MMEs and those in the hornblende granites.

\section{Discussion}

\subsection{Genetic type: highly fractionated I-type or A-type?}

Since the introduction of the terms I- and S-type granite by Chappell and White (1974), granitic rocks have commonly been divided into I-, S-, M- and Atypes according to the nature of their protolith (Pitcher, 1982, 1993). However, distinction between different types is not always straightforward. This is particularly true in the case of A-type and highly fractionated I-type granites (White and Chappell, 1983; Chappell and Stephens, 1988; Landenberger and Collins, 1996; King et al., 1997, 2001; Wu et al., 2003a). Several attempts have been made to discriminate Atypes from the others (e.g., Collins et al., 1982; Whalen et al., 1987; Sylvester, 1989; Eby, 1990, 1992). In general, A-type granites are comparatively enriched in high field strength elements (HFSEs), such as $\mathrm{Zr}, \mathrm{Nb}$, Y, REE and Ga. 

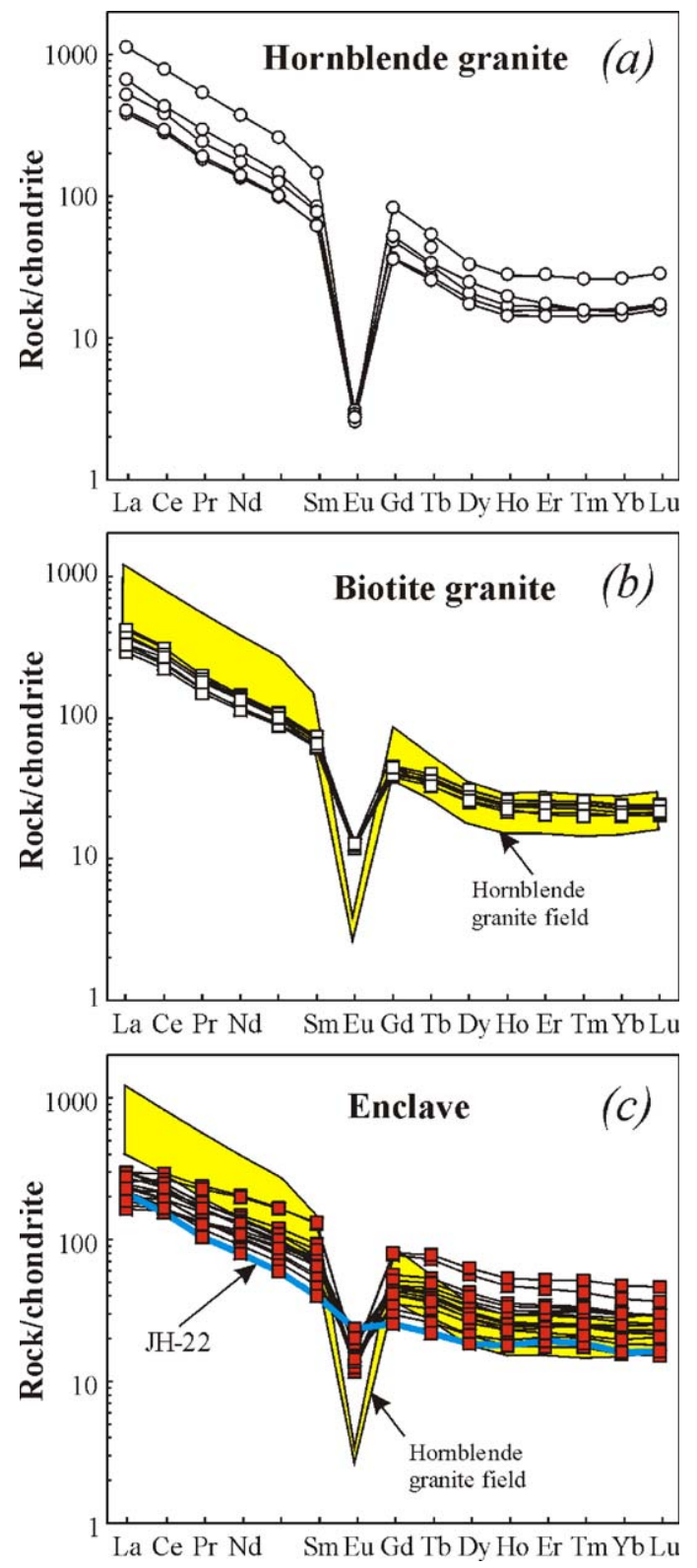

Fig. 6. Chondrite-normalized REE patterns for (a) hornblende granites, (b) biotite granites, and (c) enclaves of the Qianshan pluton. The chondrite values are from Sun and McDonough (1989).

The Qianshan granites in NE China have most characteristics of A-type granites. They have high $\mathrm{K}_{2} \mathrm{O}+\mathrm{Na}_{2} \mathrm{O}, \mathrm{Zr}$, total $\mathrm{FeO} / \mathrm{MgO}$ and $\mathrm{Ga} / \mathrm{Al}$ ratios. In the discrimination diagrams of $\mathrm{K}_{2} \mathrm{O}+\mathrm{Na}_{2} \mathrm{O}, \mathrm{Nb}$ and $\mathrm{Zr}$ vs. Ga/Al (Fig. 5a and b), they plot in the A-type granite field of Whalen et al. (1987). Furthermore, in the $\left(\mathrm{K}_{2} \mathrm{O}+\mathrm{Na}_{2} \mathrm{O}\right) / \mathrm{CaO}$ vs. $(\mathrm{Zr}+\mathrm{Nb}+\mathrm{Y}+\mathrm{Ce})$ diagram, all of them fall in the field of A-type granites (Fig. 5d). This is also confirmed by the criteria of Sylvester (1989) using the diagram of $\left(\mathrm{Al}_{2} \mathrm{O}_{3}+\mathrm{CaO}\right) /$
$\left(\mathrm{FeO}^{*}+\mathrm{Na}_{2} \mathrm{O}+\mathrm{K}_{2} \mathrm{O}\right)$ vs. $100\left(\mathrm{MgO}+\mathrm{FeO}^{*}+\mathrm{TiO}_{2}\right) /$ $\mathrm{SiO}_{2}$, which is fairly effective in discriminating alkaline granites from calc-alkaline and strongly peraluminous granites (unshown). We therefore conclude that
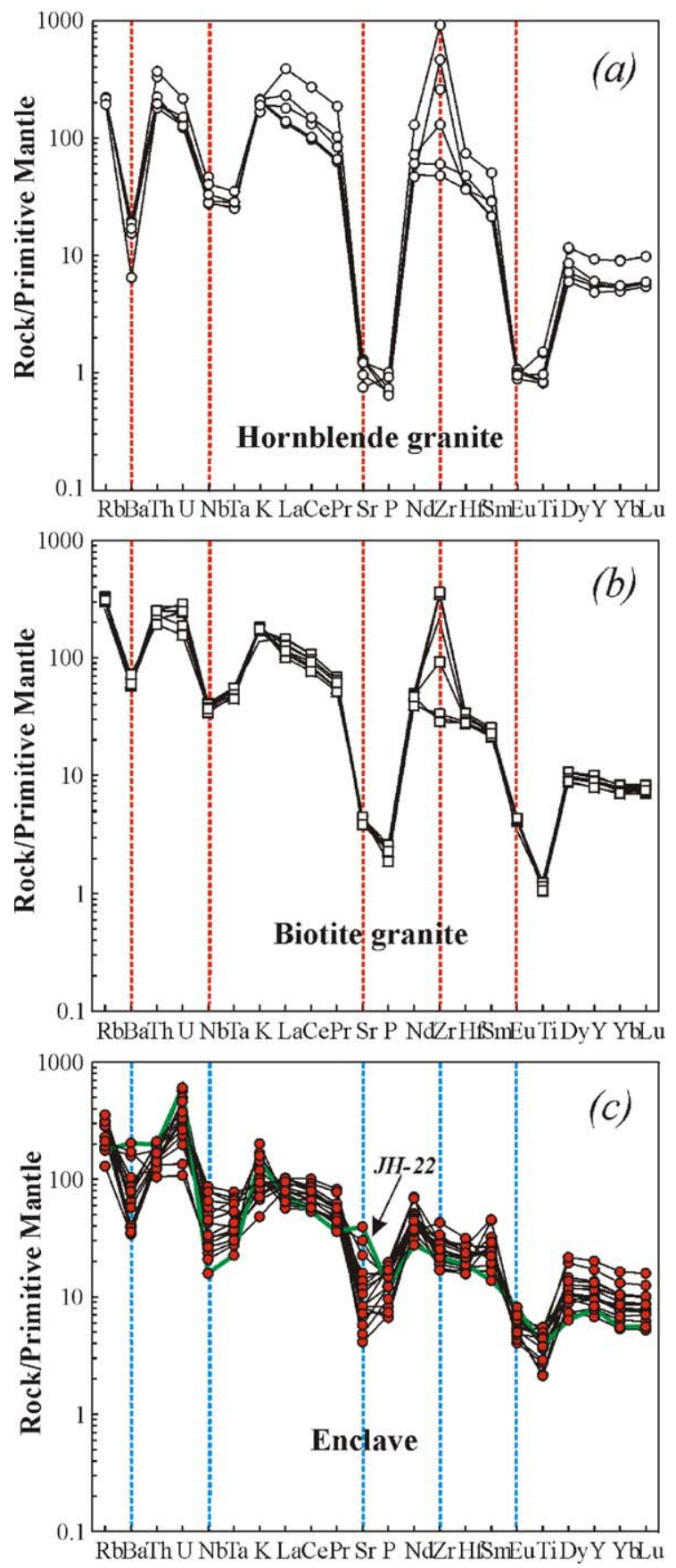

Fig. 7. Primitive mantle (PM) normalized trace element patterns, elements arranged in the order of decreasing incompatibility from left to right. (a) Hornblende granites, (b) biotite granites, and (c) enclaves of the Qianshan pluton. The PM values are from Sun and McDonough (1989). 
Table 2

$\mathrm{Rb}-\mathrm{Sr}$ and $\mathrm{Sm}-\mathrm{Nd}$ isotopic data for Qianshan granites and mafic microgranular enclaves

\begin{tabular}{|c|c|c|c|c|c|c|c|c|c|c|c|c|c|c|c|}
\hline Sample no. & Rock type & $\begin{array}{l}\mathrm{Rb} \\
(\mathrm{ppm})\end{array}$ & $\begin{array}{l}\mathrm{Sr} \\
(\mathrm{ppm})\end{array}$ & $\begin{array}{l}{ }^{87} \mathrm{Rb} / \\
{ }^{86} \mathrm{Sr}\end{array}$ & $\begin{array}{l}{ }^{87} \mathrm{Sr} / \\
{ }^{86} \mathrm{Sr}\end{array}$ & $2 \sigma$ & $\begin{array}{l}\text { Sm } \\
(\mathrm{ppm})\end{array}$ & $\begin{array}{l}\mathrm{Nd} \\
(\mathrm{ppm})\end{array}$ & $\begin{array}{l}{ }^{147} \mathrm{Sm} / \\
{ }^{144} \mathrm{Nd}\end{array}$ & $\begin{array}{l}{ }^{143} \mathrm{Nd} / \\
{ }^{144} \mathrm{Nd}\end{array}$ & $2 \sigma$ & $\begin{array}{l}\left({ }^{87} \mathrm{Sr} /\right. \\
\left.{ }^{86} \mathrm{Sr}\right)_{i}^{\mathrm{a}}\end{array}$ & $\varepsilon_{\mathrm{Nd}}(t)^{\mathrm{a}, \mathrm{b}}$ & $T_{\mathrm{DM}}^{\mathrm{a}, \mathrm{b}}$ & $f_{\mathrm{Sm} / \mathrm{Nd}}$ \\
\hline JH-07 & Biotite granite & 189 & 88.0 & 6.312 & 0.724871 & 14 & 8.87 & 50.0 & 0.1074 & 0.511897 & 5 & 0.71357 & -13.1 & 1792 & -0.45 \\
\hline JH-08 & Biotite granite & 199 & 89.9 & 6.426 & 0.724965 & 14 & 9.92 & 59.1 & 0.1015 & 0.511887 & 6 & 0.71346 & -13.2 & 1712 & -0.48 \\
\hline JH-09 & Biotite granite & 195 & 91.2 & 6.189 & 0.724698 & 13 & 8.29 & 48.2 & 0.1039 & 0.511885 & 11 & 0.71361 & -13.3 & 1752 & -0.47 \\
\hline JH-15-1 & Biotite granite & 195 & 91.8 & 6.143 & 0.724303 & 10 & 10.5 & 61.1 & 0.1042 & 0.511873 & 6 & 0.71330 & -13.6 & 1773 & -0.47 \\
\hline JH-17-1 & Biotite granite & 196 & 92.7 & 6.129 & 0.724322 & 12 & 9.00 & 50.5 & 0.1078 & 0.511909 & 9 & 0.71335 & -12.9 & 1781 & -0.45 \\
\hline FW01-162 & Biotite granite & 189 & 91.1 & 6.024 & 0.724178 & 16 & 9.63 & 59.3 & 0.0982 & 0.511897 & 10 & 0.71339 & -13.0 & 1650 & -0.50 \\
\hline JH-27 & $\begin{array}{l}\text { Hornblende } \\
\text { granite }\end{array}$ & 132 & 15.9 & 24.15 & 0.770192 & 16 & 9.21 & 60.7 & 0.0918 & 0.511743 & 6 & 0.72694 & -15.9 & 1755 & -0.53 \\
\hline JH-28 & $\begin{array}{l}\text { Hornblende } \\
\text { granite }\end{array}$ & 128 & 22.2 & 16.76 & 0.758347 & 15 & 12.3 & 94.5 & 0.0789 & 0.511739 & 16 & 0.72833 & -15.8 & 1592 & -0.60 \\
\hline JH-29 & $\begin{array}{l}\text { Hornblende } \\
\text { granite }\end{array}$ & 128 & 21.7 & 17.20 & 0.756847 & 13 & 9.01 & 62.5 & 0.0871 & 0.511738 & 5 & 0.72605 & -15.9 & 1696 & -0.56 \\
\hline JH-31 & $\begin{array}{l}\text { Hornblende } \\
\text { granite }\end{array}$ & 130 & 21.2 & 17.87 & 0.758142 & 14 & 22.2 & 175 & 0.0765 & 0.511700 & 5 & 0.72614 & -16.5 & 1607 & -0.61 \\
\hline FW01-164 & $\begin{array}{l}\text { Hornblende } \\
\text { granite }\end{array}$ & 125 & 20.2 & 18.05 & 0.753824 & 16 & 17.1 & 144 & 0.0720 & 0.511817 & 16 & 0.72151 & -14.1 & 1432 & -0.63 \\
\hline JH-10 & MME & 178 & 241 & 2.132 & 0.716645 & 12 & 17.8 & 86.6 & 0.1242 & 0.511887 & 6 & 0.71283 & -13.6 & 2143 & -0.37 \\
\hline JH-13 & MME & 206 & 316 & 1.886 & 0.714726 & 9 & 10.6 & 49.6 & 0.1291 & 0.511929 & 5 & 0.71135 & -12.9 & 2191 & -0.34 \\
\hline JH-14 & MME & 169 & 274 & 1.783 & 0.714974 & 9 & 10.9 & 60.9 & 0.1081 & 0.511915 & 14 & 0.71178 & -12.8 & 1778 & -0.45 \\
\hline JH-15-2 & MME & 177 & 83.9 & 6.112 & 0.724662 & 12 & 13.3 & 65.0 & 0.1236 & 0.511879 & 10 & 0.71372 & -13.8 & 2142 & -0.37 \\
\hline JH-16 & MME & 136 & 235 & 1.667 & 0.713782 & 7 & 9.71 & 56.5 & 0.1040 & 0.511932 & 6 & 0.71080 & -12.4 & 1688 & -0.47 \\
\hline JH-17-2 & MME & 78.9 & 173 & 1.325 & 0.715729 & 14 & 7.74 & 44.8 & 0.1045 & 0.511875 & 15 & 0.71336 & -13.5 & 1775 & -0.47 \\
\hline JH-20 & MME & 112 & 607 & 0.5358 & 0.710886 & 15 & 7.54 & 45.1 & 0.1010 & 0.511848 & 6 & 0.70993 & -14.0 & 1756 & -0.49 \\
\hline JH-22 & MME & 106 & 789 & 0.3902 & 0.710431 & 11 & 6.30 & 39.1 & 0.0976 & 0.511830 & 7 & 0.70973 & -14.3 & 1728 & -0.50 \\
\hline JH-23 & MME & 191 & 225 & 2.453 & 0.717002 & 7 & 13.3 & 66.6 & 0.1211 & 0.511912 & 11 & 0.71261 & -13.1 & 2031 & -0.38 \\
\hline JH-25 & MME & 212 & 149 & 4.131 & 0.719675 & 6 & 8.59 & 48.6 & 0.1070 & 0.511960 & 5 & 0.71228 & -11.9 & 1696 & -0.46 \\
\hline JH-30 & MME & 124 & 118 & 3.044 & 0.720206 & 11 & 9.95 & 51.5 & 0.1168 & 0.511835 & 7 & 0.71475 & -14.5 & 2061 & -0.41 \\
\hline
\end{tabular}

the Qianshan granites described in this study are Atype granites.

\subsection{Restite or magma mixing?}

The MMEs and host granites show a continuous variation in $\mathrm{SiO}_{2}$ from 56 to $71 \mathrm{wt} . \%$, producing tight linear to slightly curved trends on Harker diagrams (Fig. 4). Although this variation provides a strong argument for a genetic link between the enclaves and host granites, there are several other potential reasons for such trends, including restite unmixing, crystal fractionation of a mafic magma or two-component mixing.

In the restite unmixing model (e.g., Chappell et al., 1987; Chappell and White, 1992), MMES are thought to represent residual material (restite) which successively unmixes from the melt during the rise of a crystal mush from its source region. A critical feature of the restite model is linear chemical variations, which are observed in many plutonic suites (e.g., Chappell et al., 1987; Chappell and White, 1992; Collins, 1998). Although these features are observed in some element variation diagrams (Fig. 4), the large $\mathrm{Sr}, \mathrm{Nd}$ and $\mathrm{Hf}$ isotopic differences between enclaves and host rocks from the Qianshan pluton clearly rule out any strictly cogenetic origin. Restite derived from the source rock of the granitoids should be in isotopic equilibrium with its enclosing melt. It is therefore unlikely that the mafic microgranular enclaves from the Qianshan pluton are restitic in origin. The heterogeneous isotopic compositions of this granitoid-mafic enclave association also preclude a simple, common evolution by closed-system fractionation processes.

The enclaves from Qianshan have igneous microtextures and contain acicular apatites, which are analogous to other cases from around the world (Eichelberger, 1980; Vernon, 1984; Barbarin, 1988; Didier and Barbarin, 1991; Bonin, 2004; Yang et al., 2004b). Acicular apatite habits are common in comingled mafic pillows (Vernon et al., 1988) and have been attributed to rapid cooling, the result of mingling of small volumes of hot basalt with a cool granitic melt (Sparks and Marshall, 1986). Furthermore, the presence of megacrysts, such as $\mathrm{K}$-feldspar, in the enclaves implies that the enclaves are 


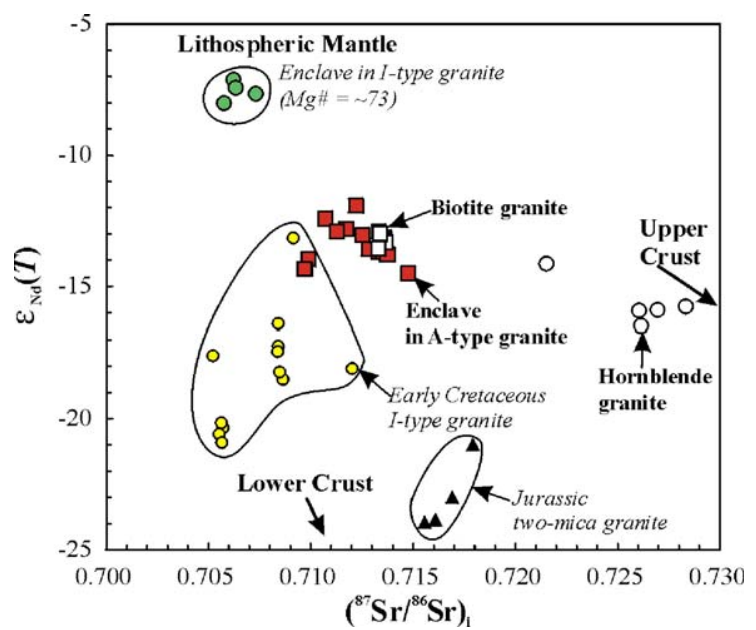

Fig. 8. $\varepsilon_{\mathrm{Nd}}(t)$ vs. $\left({ }^{87} \mathrm{Sr} /{ }^{86} \mathrm{Sr}\right)_{i}$ plot of enclaves and host granitoids of the Qianshan pluton, Liaodong Peninsula. The data for Jurassic twomica granites are from Wu et al. (2005b). The data for Early Cretaceous I-type granites and enclaves are from Yang et al. (2004b). The source end-members are from Yang et al. (2004b). The Sr and Nd isotopic compositions of lithospheric mantle represented by mafic enclaves in the I-type granites were obtained from Yang et al. (2004b).

hybrids. The fine-grained, igneous-textured mafic to intermediate enclaves from I- and A-type granites have been considered to represent remnants of a mafic component added to intermediate or felsic magma chambers (e.g., Vernon et al., 1988; Holden et al., 1987; Bédard, 1990; Didier and Barbarin, 1991; Bonin, 2004; Yang et al., 2004b). In addition, the Hf isotopic ratios are higher in the zircon cores than the rims, produced by high ${ }^{176} \mathrm{Hf} /{ }^{177} \mathrm{Hf}$ zircon growth in a low ${ }^{176} \mathrm{Hf} /{ }^{177} \mathrm{Hf}$ magmatic environment (Fig. 9). Also, the Zr contents (191 to
10,270 ppm) and $\mathrm{Zr} / \mathrm{Hf}$ ratios (37 to 448) increase with increasing $\mathrm{SiO}_{2}$ and decreasing $\mathrm{MgO}$ from the MMEs to hornblende and biotite granites (Table 1), indicating a magma mixing process in the petrogenesis of the Qianshan suite. Therefore, the enclaves represent additional components involved in the generation of the host Atype granites, a view that is compatible with the petrographic, geochemical and isotopic data.

\subsection{Sources}

Although radiogenic isotope equilibrium is attained more rapidly than chemical equilibrium in the course of magma mixing (Lesher, 1990; Poli et al., 1996), the mafic enclaves and host hornblende granites have distinct $\mathrm{Sr}$ and $\mathrm{Nd}$ isotopic compositions. This indicates that $\mathrm{Sr}$ and $\mathrm{Nd}$ isotopes were not equilibrated during mafic-felsic magma mixing and can be used to trace the sources of the mafic and felsic magmas. This is also reflected by the zircon $\mathrm{Hf}$ isotopes. Host granites are highly enriched in $\mathrm{Zr}$, up to $10,270 \mathrm{ppm}$ (Table 1), and concentrations of $\mathrm{Zr}$ in mafic enclaves range from 189 to $340 \mathrm{ppm}$, showing an increase with increasing $\mathrm{K}_{2} \mathrm{O}$, $\mathrm{Rb}$ and $\mathrm{SiO}_{2}$ (Table 1). It seems likely that there was a gain of $\mathrm{Zr}$ into the mafic magma during magma mixing, indicating that some $\mathrm{Zr}$ was transferred from the granitic magma during the mixing process. However, the variable $\mathrm{Hf}$ isotopic compositions of zircons from MMEs and host granites (Fig. 10) and higher Hf isotopic ratios of zircon cores compared to the rims (Fig. 9b) show that zircon Hf isotopes can also be used to trace the sources of mafic and felsic magmas.

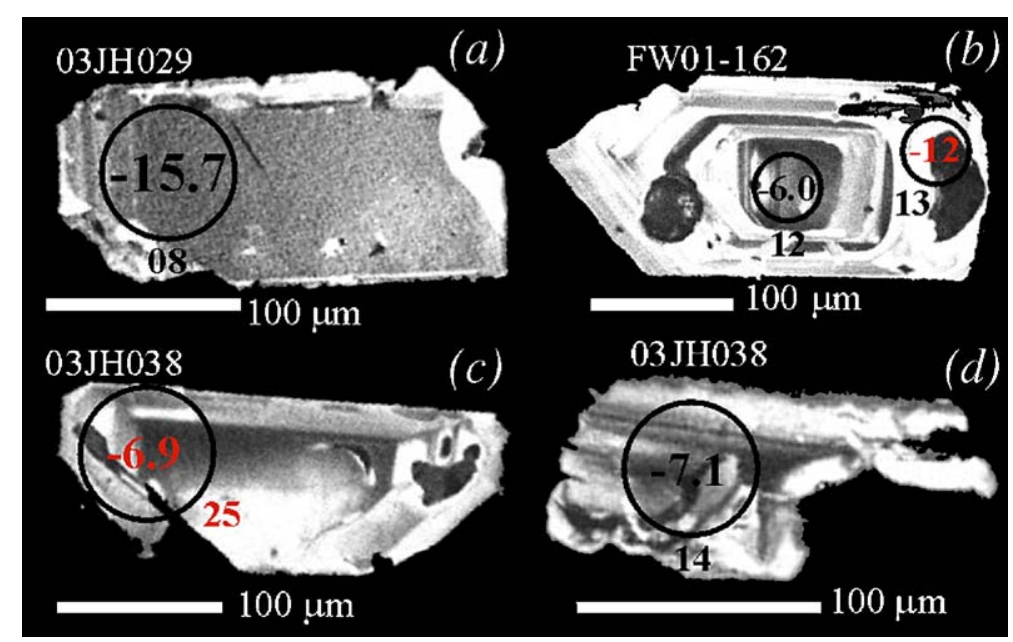

Fig. 9. Cathodoluminescence (CL) images of representative zircons from enclaves and host Qianshan granites. Circles indicate the location of LAMC-ICPMS Hf analyses and the numbers in circles refer to the $\varepsilon_{\mathrm{Hf}}(t)$ values. Spot numbers are labelled near the circles. (a) CL image of zircon in hornblende granite; (b) CL image of zircon in biotite granite; and (c) and (d) CL images of zircons in enclaves. All $\varepsilon_{\mathrm{Hf}}(t)$ values of enclave and host granites were calculated at $\sim 126 \mathrm{Ma}$, the emplacement age of the Qianshan pluton. 

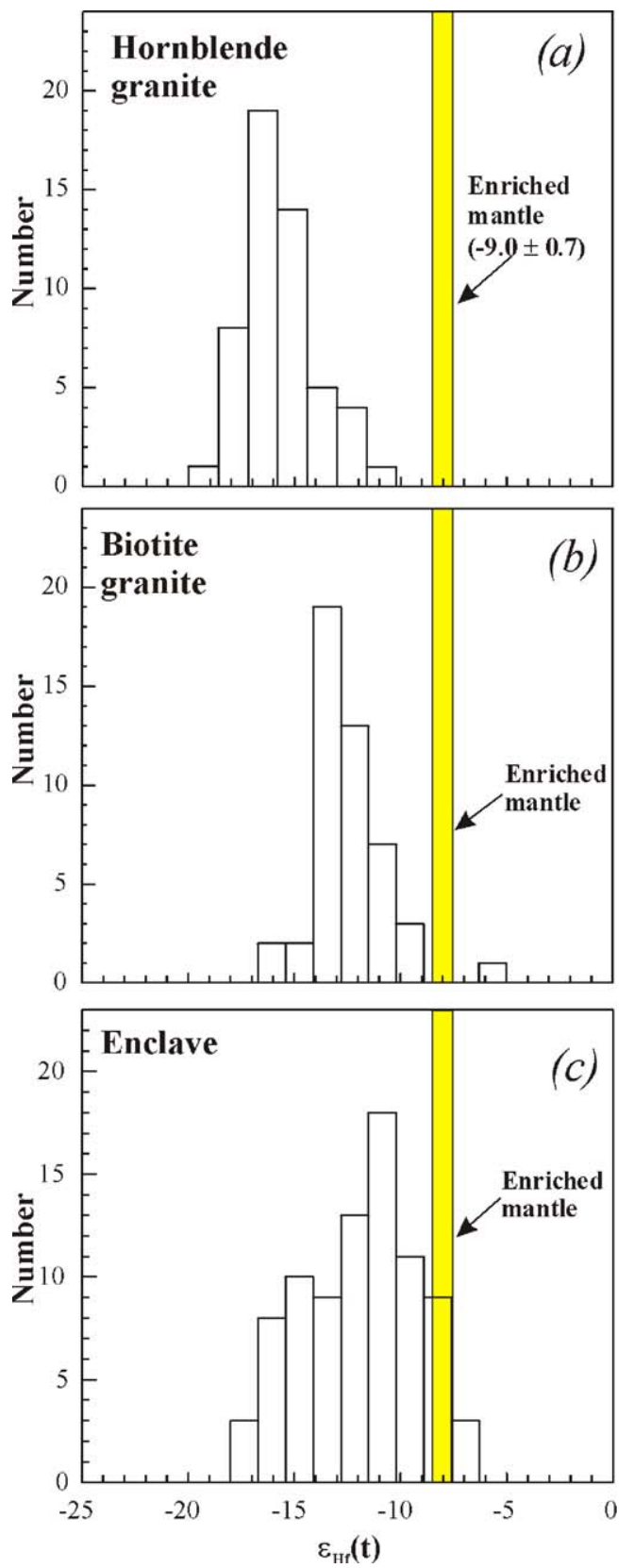

Fig. 10. Histograms of $\varepsilon_{\mathrm{Hf}}(t)$ values for zircons in (a) hornblende granites, (b) biotite granites and (c) enclaves. All $\varepsilon_{\mathrm{Hf}}(t)$ values of enclaves and host granites were calculated at $\sim 126 \mathrm{Ma}$, the emplacement ages of the Qianshan pluton. The $\varepsilon_{\mathrm{Hf}}(t)$ value $(-9.0 \pm 0.7)$ for enriched mantle is represented by mafic dykes obtained by Yang (2005, unpublished data).

In Fig. 8, the $\mathrm{Sr}$ and $\mathrm{Nd}$ isotopic data from the Qianshan granites and their mafic enclaves may be interpreted as a result of two-component mixing between mafic $\left[\left({ }^{87} \mathrm{Sr} /{ }^{86} \mathrm{Sr}\right)_{i}<0.7097\right.$, and $\left.\varepsilon_{\mathrm{Nd}}(t)>-11.9\right]$ and felsic $\left[\left({ }^{87} \mathrm{Sr} /{ }^{86} \mathrm{Sr}\right)_{i}>0.7283\right.$, and $\left.\varepsilon_{\mathrm{Nd}}(t)<-16.5\right]$ isotopically evolved end-members, which are represented by the isotopic compositions of the mafic dykes or mafic enclaves in the Gudaoling I-type granites near the Qianshan pluton (Yang et al., 2004b) and hornblende granites, respectively.

The hornblende granites have high initial ${ }^{87} \mathrm{Sr} /{ }^{86} \mathrm{Sr}$ ratios, high $\mathrm{Rb} / \mathrm{Sr}$ ratios and negative $\varepsilon_{\mathrm{Nd}}(t)$ values at $126 \mathrm{Ma}$, indicating that they were derived from a crustal source with more radiogenic $\mathrm{Sr}$ and less radiogenic $\mathrm{Nd}$ isotopic ratios. Although it has been proposed that A-type granites originated from the partial melting of F- and/or Cl-enriched dry, granulitic (or charnockitic) residual material from which a granitoid melt was previously extracted (Collins et al., 1982; Clemens et al., 1986; Creaser et al., 1991; King et al., 1997), this model cannot explain the geochemical characteristics of the hornblende granites. In the Liaodong Peninsula, the synchronous I-type granites have geochemical and isotopic features distinct from the A-type granites (Yang et al., 2004b), indicating they cannot be derived from a common source. Geochemical characteristics of the hornblende granites, including low $\mathrm{Al}$ and $\mathrm{Ca}$ contents (Fig. 4) and high $\mathrm{FeO} * / \mathrm{MgO}$ and $\left(\mathrm{Na}_{2} \mathrm{O}+\mathrm{K}_{2} \mathrm{O}\right) / \mathrm{Al}_{2} \mathrm{O}_{3}$ ratios compared to calc-alkaline granitoids, the enrichment in high field strength elements, such as $\mathrm{Zr}, \mathrm{Nb}$, and $\mathrm{Y}$, as well as the $\mathrm{Ga} / \mathrm{Al}$ ratio (Fig. 5), and the depletion in $\mathrm{Eu}$ and $\mathrm{Sr}$, are all consistent with the results from experimental melts (Skjerlie and Johnston, 1993; Patiño Douce, 1995, 1997, 1999) produced by dehydration melting of calc-alkaline granitoids at low pressure ( $P \leq 4$ kbar). Therefore, dehydration melting of hornblende and biotite-bearing assemblages with a plagioclase-rich residual assemblage in the shallow crust (at depths of $15 \mathrm{~km}$ or less) is a likely origin for the hornblende granite, as evidenced by the fact that the hornblende granites have high $\mathrm{Rb} / \mathrm{Sr}$ ratios and concave-up REE patterns with strongly negative Eu anomalies (Fig. $6 a)$. However, attaining the required melting temperatures $\left(>900^{\circ} \mathrm{C}\right)$ so close to the Earth's surface requires the participation of hot mafic magmas in the origin of Atype granites. The variable $\mathrm{Sr}, \mathrm{Nd}$ and zircon $\mathrm{Hf}$ isotopic compositions indicate that a mafic magma with high $\mathrm{Nd}$ and $\mathrm{Hf}$ isotopic ratios, corresponding to the parental magma to the MMEs, is involved in the origin of hornblende granites. Thus, we propose that the hornblende granites were mainly derived from partial melting of a hornblende- and/or biotite-bearing source, with a plagioclase-rich residual assemblage. The with force was the emplacement of mafic magma into the upper crust, with which the granitic magmas interacted.

The source of the biotite granites (at $126 \mathrm{Ma}$ ) had more radiogenic $\mathrm{Nd}$ isotopic ratios and much less ra- 
diogenic ${ }^{87} \mathrm{Sr} /{ }^{86} \mathrm{Sr}$ ratios than the hornblende granites, ruling out magma derivation solely by partial melting of an upper crustal source. Therefore, sources other than the upper crust must also be involved. It is unlikely that the lower crust was the source for the biotite granites, because the lower crust beneath the Liaodong Peninsula has much less radiogenic $\mathrm{Nd}\left(\varepsilon_{\mathrm{Nd}}(t)=-17\right.$ to -20$)$ and $\mathrm{Sr}$ (initial ${ }^{87} \mathrm{Sr} /{ }^{86} \mathrm{Sr}=0.7055-0.7116$ ) isotopic ratios (Yang et al., 2004b). Neither derivation from lower crust or upper crust nor any combination of the two can account for the isotopic composition of the Qianshan biotite granites. Importantly, the biotite granites have similar whole rock $\mathrm{Sr}$ and $\mathrm{Nd}$ and zircon $\mathrm{Hf}$ isotopic compositions to the mafic enclaves, indicating they were derived from a common source.

Mafic enclaves have relatively higher Mg-numbers (19.7-54.0) than the host granites. Sample JH-22, with the highest $\mathrm{Mg}$-number (54.0), has the lowest silica content $\left(\mathrm{SiO}_{2}=60 \%\right)$ without negative $\mathrm{Ba}, \mathrm{Sr}$ and $\mathrm{Eu}$ anomalies, indicating that a component with a high $\mathrm{MgO}$ content was involved in the genesis of this enclave. Although it has a highly negative $\varepsilon_{\mathrm{Nd}}(t)$ value $(-14.3)$, this is because isotopic equilibrium was attained more rapidly than chemical equilibrium in the course of magma mixing (Lesher, 1990; Poli et al., 1996). The source component should have lower $\mathrm{SiO}_{2}$ concentrations, higher $\mathrm{MgO}$, more radiogenic $\mathrm{Nd}$ isotopic ratios $\left(\varepsilon_{\mathrm{Nd}}(t)>-12\right)$ and less radiogenic ${ }^{87} \mathrm{Sr} /{ }^{86} \mathrm{Sr}$ ratios $(<0.7097)$ than either the granites or the mafic enclaves. Such geochemical features and isotopic compositions are similar to those of magmas derived from lithospheric mantle, represented by mafic enclaves hosted by Early Cretaceous I-type granites or Early Cretaceous mafic dykes in the Liaodong Peninsula (Yang et al., 2004b). The enclaves in I-type granites and mafic dykes have low to moderate $\mathrm{SiO}_{2}$ contents (51-57\%), high Mg-numbers (64-72), initial ${ }^{87} \mathrm{Sr} /{ }^{86} \mathrm{Sr}$ ratios of $0.7058-0.7073$, $\varepsilon_{\mathrm{Nd}}(125 \mathrm{Ma})$ of -7.2 to $-8.0, \varepsilon_{\mathrm{Hf}}(125 \mathrm{Ma})$ of -2.1 , and are enriched in LILEs and LREEs and depleted in high field strength elements (HFSEs), indicating they were derived from enriched lithospheric mantle (Yang et al., 2004b). However, mafic enclaves in the Qianshan granites have variable major and trace element contents (Table 1, Figs. 4 and 7) and are enriched in $\mathrm{K}_{2} \mathrm{O}, \mathrm{Rb}$ and $\mathrm{Zr}$, indicating mixing with granitic magmas.

\subsection{Genesis of the Qianshan pluton}

The hornblende granites were derived by partial melting of upper crust at low pressure, but involving lithospheric mantle-derived materials. They plot close to the theoretical upper crust end-member in the
$\varepsilon_{\mathrm{Nd}}(t)$ vs. $\left({ }^{87} \mathrm{Sr} /{ }^{86} \mathrm{Sr}\right)_{i}$ diagram (Fig. 8), whereas, the biotite granites and mafic enclaves plot on the binary mixing line between lithospheric mantle and upper crust. Two-component mixing calculations suggest that as much as $10 \%$ to $20 \%$ hybridization with mantle-derived melts accounts for the $\mathrm{Sr}$ and $\mathrm{Nd}$ isotopic
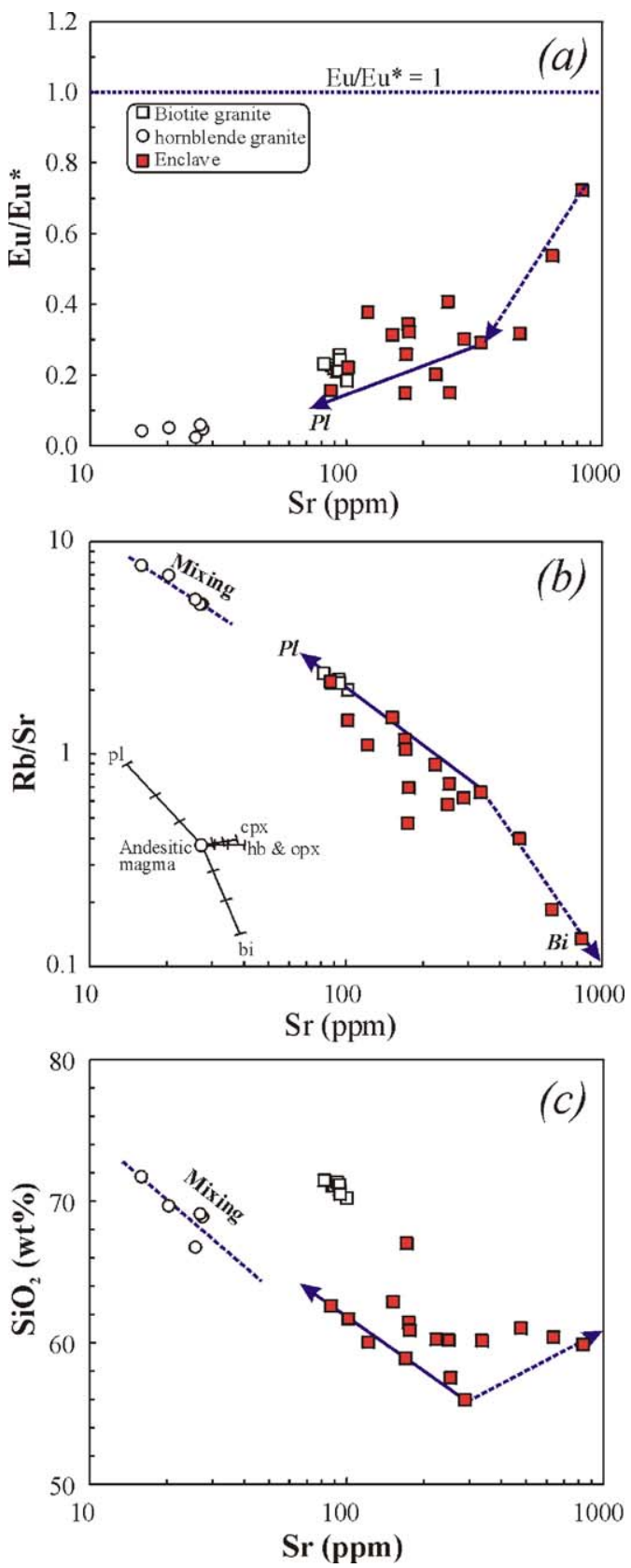

Fig. 11. (a) $\mathrm{Eu} / \mathrm{Eu}^{*}$, (b) $\mathrm{Rb} / \mathrm{Sr}$ and (c) $\mathrm{SiO}_{2}$ vs. Sr diagrams showing plagioclase- and biotite-dominated fractionation in the evolution of an evolved mafic magma. Partition coefficients are from Rollinson (1993). Symbols are: $\mathrm{pl}=$ plagioclase; $\mathrm{hb}=$ hornblende; $\mathrm{cpx}=$ clinopyroxene; opx $=$ orthopyroxene; $\mathrm{bi}=$ biotite. 
heterogeneity of the A-type granites and the mafic enclaves.

Besides magma mixing, all MMEs and biotite granites plot on the trends of biotite and plagioclase fractionation in the $\mathrm{Eu} / \mathrm{Eu}^{*}, \mathrm{Rb} / \mathrm{Sr}$ and $\mathrm{SiO}_{2}$ vs. $\mathrm{Sr}$ diagrams (Fig. 11). This indicates that the biotite granites were generated by fractionation of both biotite and plagioclase in a mafic magma or formed as an evolved mafic magma derived from an enriched lithospheric mantle source.

Based on the petrography, major and trace element geochemistry and $\mathrm{Sr}, \mathrm{Nd}$ and zircon $\mathrm{Hf}$ isotopic compositions of mafic microgranular enclaves and their host granites, a complex, multi-stage process involving magma mixing, crystal fractionation and partial melting of upper crustal source rocks is proposed to explain the formation of the Qianshan A-type granites.

In the Liaodong Peninsula, the Qianshan pluton is coeval with formation of Early Cretaceous metamorphic core complexes (120 to $110 \mathrm{Ma}$ ), indicating an extensional regime at this time. This most likely resulted from lithospheric thinning and possible subduction of the Paleo-Pacific ocean plate (Menzies et al., 1993; Griffin et al., 1998; Wu et al., 2003b; Yang et al., 2003; Gao et al., 2004; Wu et al., 2005a). Furthermore, the formation of hornblende granites by partial melting of upper crust indicates a high temperature in the upper crust, evidenced by the formation of large-scale gold mineralization in the Liaodong Peninsula which was a result of thermal fluid activity at a shallow crustal level (Yang et al., 2003). Thus, we suggest that the Qianshan pluton was the result of lithospheric thinning that led to intensive underplating of mantle-derived magma and a high degree of crustal melting, thereby producing strong magma mixing and hybrid granitoids in a largely extensional tectonic setting.

\section{Concluding remarks}

Mafic microgranular enclaves and host A-type granites of the Qianshan pluton in the Liaodong Peninsula of NE China provide direct evidence of the involvement of mantle-derived magmas in A-type granitoid genesis. The geochemical characteristics and isotopic compositions of the Qianshan A-type granites and their mafic enclaves imply that they were generated not via partial melting of lower crustal granulitic residues or by fractionation of alkali basalts, but by mixing of enriched lithospheric mantle-derived magma with upper crustal-derived magmas.

Basic magmas were emplaced penecontemporaneously with A-type granitoids and were contaminated during their ascent by crustal materials. Although it is difficult to deduce precise estimates for the isotopic signature of the basaltic component, the available data suggest that magmas with high $\mathrm{MgO}$, negative $\varepsilon_{\mathrm{Nd}}(t)$ and $\varepsilon_{\mathrm{Hf}}(t)$, and high $\left({ }^{87} \mathrm{Sr} /{ }^{86} \mathrm{Sr}\right)$, i.e., partial melts of an enriched lithospheric mantle and its evolved products, were involved in the hybridization process. Hornblende granites with high $\mathrm{Rb} / \mathrm{Sr}$ and $\left({ }^{87} \mathrm{Sr} /{ }^{86} \mathrm{Sr}\right)_{i}$ ratios and negative $\varepsilon_{\mathrm{Nd}}(t)$ and $\varepsilon_{\mathrm{Hf}}(t)$ values are interpreted to represent an upper crustal-derived magma.

The Qianshan plutonic association thus illustrates that hybridization of mantle- and crustal-derived magmas is a complex, multiple-stage process involving several different mechanisms (magma mixing, crystal fractionation, and crustal anatexis) which all operated together during Late Mesozoic extension in the Liaodong Peninsula of NE China.

\section{Acknowledgements}

We thank Dr. Fu-Kun Chen and Chao-Feng Li for helping with $\mathrm{Sr}$ and $\mathrm{Nd}$ isotopic analyses, Dr. Qian Mao and Yu-Guang Ma for helping with CL imaging and Dr. Lie-Wen Xie for helping with zircon Hf isotopic analyses. We thank Stephen Foley (chief editor), Boris A. Litvinovsky and an anonymous reviewer for their constructive comments. This study was supported by grants 40325006, 40203005 from the National Natural Science Foundation of China and "Funds for Hundred Outstanding Talents Plan".

\section{Appendix A. Supplementary data}

Supplementary data associated with this article can be found, in the online version, at doi:10.1016/j.lithos. 2005.10.002.

\section{References}

Barbarin, B., 1988. Field evidence for successive mixing and mingling between the Piolard diorite and the Saint-Julien-La-Vetre monzogranite (Nord-Forez Massif Central, France). Can. J. Earth Sci. 25, 49-59.

Bédard, J., 1990. Enclaves from the A-type granite of the Mégantic Complex, White Mountain magma series: clues to granite magmagenesis. J. Geophy. Res. 95, 17797-17819.

Black, R., Liegeois, J.P., 1993. Cratons, mobile belts, alkaline rocks and continental lithospheric mantle; the Pan-African testimony. J. Geol. Soc. (Lond.) 150, 89-98.

Blichert-Toft, J., Albarede, F., 1997. The Lu-Hf isotope geochemistry of chondrites and the evolution of the mantle-crust system. Earth Planet. Sci. Lett. 148, 243-258.

Bonin, B., 2004. Do coeval mafic and felsic magmas in post-collisional to within-plate regimes necessarily imply two contrasting, mantle and crust, sources? A review. Lithos 78, 1-24. 
Chappell, B.W., White, A.J.R., 1974. Two contrasting granite types. Pac. Geol. 8, 173-174.

Chappell, B.W., Stephens, W.E., 1988. Origin of infracrustal (I-type) granite magmas. Trans. R. Soc. Edinb. Earth Sci. 79, 71-86.

Chappell, B.W., White, A.J.R., 1992. I- and S-type granites in the Lachlan Fold Belt. Trans. R. Soc. Edinb. Earth Sci. 83, 1-26.

Chappell, B.W., White, A.J.R., Wyborn, D., 1987. The importance of residual source material (restite) in granite petrogenesis. J. Petrol. $28,1111-1138$.

Clemens, J.D., Holloway, J.R., White, A.J.R., 1986. Origin of an Atype granite: experimental constraints. Am. Mineral. 71, 317-324.

Collins, W.J., 1998. Evaluation of petrogenetic models for Lachlan Fold Belt granitoids: implications for crustal architecture and tectonic models. Aust. J. Earth Sci. 45, 483-500.

Collins, W.J., Beams, S.D., White, A.J.R., Chappell, B.W., 1982. Nature and origin of A-type granites with particular reference to southeastern Australia. Contrib. Mineral. Petrol. 80, 189-200.

Creaser, R.A., Price, R.C., Wormald, R.J., 1991. A-type granites revisited: assessment of a residual-source model. Geology 19, $163-166$.

Didier, D., Barbarin, B., 1991. Enclaves and Granite Petrology, Developments in Petrology. Elsevier Science Publications, Amsterdam. 1-625 pp.

DePaolo, D.J., 1981. Neodymium isotopes in the Colorado Front Range and crust-mantle evolution in the Proterozoic. Nature 291, 193-196.

Eby, G.N., 1990. A-type granitoids; a review of their occurrence and chemical characteristics and speculations on their petrogenesis. Lithos 26, 115-134.

Eby, G.N., 1992. Chemical subdivision of the A-type granitoids; petrogenetic and tectonic implications. Geology 20, 641-644.

Eichelberger, J.C., 1980. Vesiculation of mafic magma during replenishment of silicic magma reservoirs. Nature 288, 446-450.

Gao, S., Rudnick, R.L., Yuan, H.L., Liu, X.M., Liu, Y.S., Xu, W.L., Ling, W.L., Ayers, J., Wang, X.C., Wang, Q.H., 2004. Recycling lower continental crust in the North China Craton. Nature 432, 892-897.

Goolaerts, A., Mattielli, N., de Jong, J., Weis, D., Scoates, J.S., 2004. $\mathrm{Hf}$ and $\mathrm{Lu}$ isotopic reference values for the zircon standard 91500 by MC-ICP-MS. Chem. Geol. 206, 1-9.

Griffin, W.L., Zhang, A., O'Reilly, S.Y., Ryan, C.G., 1998. Phanerozoic evolution of the lithosphere beneath the Sino-Korean Craton. In: Flower, M.F.J., Chung, S.L., Lo, C.H., Lee, T.Y. (Eds.), Mantle Dynamics and Plate Interaction in East Asia, Geodynamics Series, vol. 27, pp. 107-126.

Griffin, W.L., Pearson, N.J., Belousova, E., Jackson, S.E., van Achterbergh, E., O'Reilly, S.Y., Shee, S.R., 2000. The Hf isotope composition of cratonic mantle: LAM-MC-ICPMS analysis of zircon megacrysts in kimberlites. Geochim. Cosmochim. Acta 64, $133-147$.

Holden, P., Halliday, A.N., Stepens, W.E., 1987. Neodymium and strontium isotope content of microdiorite enclaves points to mantle input to granitoid production. Nature 330, 53-56.

Jacobsen, S.B., Wasserburg, G.J., 1980. Sm-Nd isotopic evolution of chondrites. Earth Planet. Sci. Lett. 50, 139-155.

Kerr, A., Fryer, B.J., 1993. Nd isotopic evidence for crust-mantle interaction in the generation of A-type granitoid suites in Labrador, Canada. Chem. Geol. 104, 39-60.

King, P.L., White, A.J.R., Chappell, B.W., Allen, C.M., 1997. Characterization and origin of aluminous A-type granites from the Lachlan Fold Belt, southeastern Australia. J. Petrol. 38, $371-391$.
King, P.L., Chappell, B.W., Allen, C.M., White, A.J.R., 2001. Are Atype granites the high-temperature felsic granites? Evidence from fractionated granites of the Wangrah Suite. Aust. J. Earth Sci. 48, $501-514$.

LBGMR (Liaoning Bureau of Geology and Mineral Resources), 1986. Regional Geology of Liaoning Province (in Chinese with English abstract). Geological Publishing House, Beijing.

Landenberger, B., Collins, W.J., 1996. Derivation of A-type granites from a dehydration charnockitic lower crust: evidence from the Chaelundi complex, eastern Australia. J. Petrol. 37, 145-170.

Lee, C.Y., Tsai, J.H., Ho, H.H., Yang, T.F., Chung, S.L., Chen, C.H., 1997. Quantitative analysis in rock samples by an X-ray fluorescence spectrometer (I) major elements. Program with Abstracts of 1997 Annual Meeting of Geological Society of China, Taipei, pp. 418-420 (in Chinese).

Lesher, C.E., 1990. Decoupling of chemical and isotopic exchange during magma mixing. Nature 344, 235-237.

Liu, D.Y., Nutman, A.P., Compston, W., Wu, J.S., Shen, Q.H., 1992. Remnants of 3800 Ma crust in the Chinese part of the SinoKorean Craton. Geology 20, 339-342.

Litvinovsky, B.A., Jahn, B.-M., Zanvilevich, A.N., Saunders, A., Poulain, S., Kuzmin, D.V., Reichow, M.K., Titov, A.V., 2002. Petrogenesis of syenite-granite suites from the Bryansky Complex (Transbaikalia, Russia): implications for the origin of A-type granitoid magmas. Chem. Geol. 189, 105-133.

Lu, X.P., Wu, F.Y., Lin, J.Q., Sun, D.Y., Zhang, Y.B., Guo, C.L., 2004. Geochronology of the early Precambrian granitic magmatism in the southern Liaodong Peninsula (in Chinese with English abstract). China J. Geol. 39, 123-138.

Menzies, M.A., Fan, W.M., Zhang, M., 1993. Palaeozoic and Cenozoic lithoprobes and the loss of $>120 \mathrm{~km}$ of Archean lithosphere, Sino-Korean Craton, China. In: Prichard, H.M., et al., (Eds.), Magmatic Processes and Plate Tectonics, Geol. Soc. London Spec. Pub., vol. 76, pp. 71-81.

Mingram, B., Trumbull, R.B., Littman, S., Gerstenberger, H., 2000. A petrogenetic study of anorogenic felsic magmatism in the Cretaceous Paresis ring complex, Namibia: evidence for mixing of crust and mantle-derived components. Lithos 54, 1-22.

Mushkin, A., Navon, O., Halicz, L., Hartmann, G., Stein, M., 2003. The petrogenesis of A-type magmas from the Amram Massif, southern Israel. J. Petrol. 44, 815-832.

Patiño Douce, A.E., 1995. Dehydration-melting of biotite gneiss and quartz amphibolite from 3 to 15 kbar. J. Petrol. 36, 707-738.

Patiño Douce, A.E., 1997. Generation of metaluminous A-type granites by low-pressure melting of calc-alkaline granitoids. Geology $25,743-746$.

Patiòo Douce, A.E., 1999. What do experiments tell us about the relative contribution of crust and mantle to the origin of granitic magmas? In: Castro, A., Fernández, C., Vignersse, J.L. (Eds.), Understanding Granites: Integrating New and Classical Techniques, Geol. Soc. Lond. Spec. Publ., vol. 168, pp. 55-76.

Pitcher, W.S., 1982. Granite type and tectonic environment. In: Hsu, K.J. (Ed.), Mountain Building Processes. Academic Press, London, pp. 19-40.

Pitcher, W.S., 1993. The Nature and Origin of Granite. Blackie Academic and Professional, London. 312 pp.

Poli, G., Tommasini, S., Halliday, A.N., 1996. Trace elements and isotopic exchange during acid-basic magma interaction processes. Trans. R. Soc. Edinb. Earth Sci. 87, 225-232.

Rollinson, H.R., 1993. Using Geochemical Data: Evaluation, Presentation, Interpretation. Longman Singapore Publishers (Pte) Ltd., Singapore. 
Skjerlie, K.P., Johnston, A.D., 1993. Vapor-absent melting at $10 \mathrm{kbar}$ of a biotite- and amphibole-bearing tonalitic gneiss: implications for the generation of A-type granites. Geology 20, 263-266.

Soderlund, U., Patchett, P.J., Vervoort, J.D., Isachsen, C.E., 2004. The ${ }^{176} \mathrm{Lu}$ decay constant determined by $\mathrm{Lu}-\mathrm{Hf}$ and $\mathrm{U}-\mathrm{Pb}$ isotope systematics of Precambrian mafic intrusions. Earth Planet. Sci. Lett. 219, 311-324

Sparks, R.S.J., Marshall, L., 1986. Thermal and mechanical constraints on mixing between mafic and silicic magmas. J. Volcanol. Geotherm. Res. 29, 99-124.

Song, B., Nutman, A.P., Liu, D.Y., Wu, J.S, 1996. 3800-2500 Ma crustal evolution in the Anshan area of Liaoning Province, northeastern China. Precambrian Res. 78, 79-94.

Sun, S.S., McDonough, W.F., 1989. Chemical and isotopic systematics of oceanic basalts: implications for mantle composition and processes. In: Saunders, A.D., Norry, M.J. (Eds.), Magmatism in the Ocean Basins, Geol. Soc. London Special Publ., vol. 42, pp. 313-345.

Sylvester, P.J., 1989. Post-collisional alkaline granites. J. Geol. 97, 261-280.

Turner, S.P., Foden, J.D., Morrison, R.S., 1992. Derivation of some A-type magmas by fractionation of basaltic magma; an example from the Padthaway Ridge, South Australia. Lithos 28, 151-179.

Vernon, R.H., 1984. Microgranitoid enclaves: globules of hybrid magma quenched in a plutonic environment. Nature 304, $438-439$.

Vernon, R.H., Etheridge, M..E., Wall, V.J., 1988. Shape and microstructure of microgranitoid enclaves: indicators of magma mingling and flow. Lithos 22, 1-11.

Wang, K.L., Chung, S.L., O’Reilly, S.Y., Sun, S.S., Shinjo, R., Chen, C.H., 2004. Geochemical constraints for the genesis of postcollisional magmatism and the geodynamic evolution of the northern Taiwan region. J. Pet. 45, 975-1011.

Wang, H.Z., Mo, X.X., 1996. An outline of the tectonic evolution of China. Episodes 18, 6-16.

Whalen, J.B., Currie, K.L., Chappell, B.W., 1987. A-type granites: geochemical characteristics, discrimination and petrogenesis. Contrib. Mineral. Petrol. 95, 407-419.

Wickham, S.M., Alberts, A.D., Litvinovsky, B.A., Bindeman, I.N., Schauble, E.A., 1996. A stable isotope study of anorogenic magmatism in East Central Asia. J. Petrol. 37, 1063-1095.

Wiebe, R.A., Smith, D., Sturm, M., King, E.M., Seckler, M.S., 1997. Enclaves in the Cadillac Mountain granite (coastal Maine): samples of hybrid magma from the base of the chamber. J. Petrol. 38, $393-423$.
White, A.J.R., Chappell, B.W., 1983. Granitoid types and their distribution in the Lachlan Fold Belt, southeastern Australia. In: Roddick, J.A. (Ed.), Circum-Pacific Plutonic Terranes, Geol. Soc. Am., Mem., vol. 159, pp. 21-34.

Woodhead, J., Hergt, J., Shelley, M., Eggins, S., Kemp, R., 2004. Zircon Hf-isotope analysis with an excimer laser, depth profiling, ablation of complex geometries, and concomitant age estimation. Chem. Geol. 209, 121-135.

Wu, F.Y., Jahn, B.M., Wilde, S.A., Lo, C.H., Yui, T.F., Lin, Q., Ge, W.C., Sun, D.Y., 2003a. Highly fractionated I-type granites in NE China (I): geochronology and petrogenesis. Lithos 66, 241-273.

Wu, F.Y., Walker, R.J., Ren, X.W., Sun, D.Y., Zhou, X.H., 2003b. Osmium isotopic constraints on the age of lithospheric mantle beneath northeastern China. Chem. Geol. 196, 107-129.

Wu, F.Y., Lin, J.Q., Wilde, S.A., Zhang, X.O., Yang, J.H., 2005a. Nature and significance of the Early Cretaceous giant igneous event in eastern China. Earth Planet. Sci. Lett. 233, 103-119.

Wu, F.Y., Yang, J.H., Wilde, S.A., Zhang, X.O., 2005b. Geochronology, petrogenesis and tectonic implications of the Jurassic granites in the Liaodong Peninsula, NE China. Chem. Geol. 221, $127-156$.

Yang, J.H., Wu, F.W., Wilde, S.A., 2003. A review of the geodynamic setting of large-scale Late Mesozoic gold mineralization in the North China Craton: an association with lithospheric thinning. Ore Geol. Rev. 23, 125-152.

Yang, J.H., Wu, F.Y., Zhang, Y.B., Zhang, Q., Wilde, S.A., 2004a. Identification of Mesoproterozoic zircons in a Triassic dolerite from the Liaodong Peninsula, northeast China. Chin. Sci. Bull. 49 , $1958-1962$.

Yang, J.H., Wu, F.Y., Chung, S.L., Chu, M.F., Wilde, S.A., 2004b. Multiple sources for the origin of granites: geochemical and $\mathrm{Nd} / \mathrm{Sr}$ isotopic evidence from the Gudaoling granite and its mafic enclaves, northeast China. Geochim. Cosmochim. Acta 68, $4469-4483$.

Yang, J.H., Chung, S.L., Wilde, S.A., Wu, F.Y., Chu, M.F., Lo, C.H., Fan, H.R., 2005. Petrogenesis of post-orogenic syenites in the Sulu Orogenic Belt, East China: geochronological, geochemical and $\mathrm{Nd}-\mathrm{Sr}$ isotopic evidence. Chem. Geol. 214, 99-125.

Xu, P., Wu, F.Y., Xie, L.W., Yang, Y.H., 2004. Hf isotopic compositions of the standard zircons for $\mathrm{U}-\mathrm{Pb}$ dating. Chin. Sci. Bull. 49, $1642-1648$

Zhao, G.C., Wilde, S.A., Cawood, P.A., Sun, M., 2001. Archean blocks and their boundaries in the North China Craton: lithological, geochemical, structural and $P-T$ path constraints and tectonic evolution. Precambrian Res. 107, 45-73. 